\title{
Reversibility of a quantum channel: general conditions and their applications to Bosonic linear channels
}

\author{
M.E. Shirokov* \\ Steklov Mathematical Institute, Moscow, Russia
}

\begin{abstract}
The method of complementary channel for analysis of reversibility (sufficiency) of a quantum channel with respect to families of input states (pure states for the most part) are considered and applied to Bosonic linear (quasi-free) channels, in particular, to Bosonic Gaussian channels.

The obtained reversibility conditions for Bosonic linear channels have clear physical interpretation and their sufficiency is also shown by explicit construction of reversing channels. The method of complementary channel gives possibility to prove necessity of these conditions and to describe all reversed families of pure states in the Schrodinger representation.
\end{abstract}

Some applications in quantum information theory are considered.

Conditions for existence of discrete classical-quantum subchannels and of completely depolarizing subchannels of a Bosonic linear channel are obtained in the Appendix.

\section{Contents}

I Introduction

II Preliminaries

III General conditions for reversibility

IV Reversibility of Bosonic linear channels

IV.1 Reversibility conditions . . . . . . . . . . . . . . . . . . . . . 12

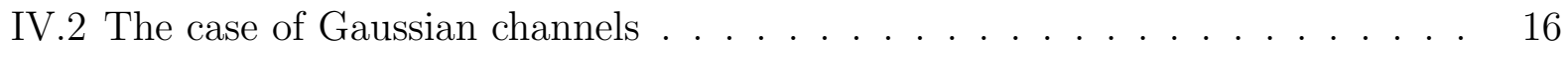

IV.3 Explicit forms of reversed families . . . . . . . . . . . . . . . . 17

V Applications

*email:msh@mi.ras.ru 


\section{Introduction}

Reversibility (sufficiency) of a quantum channel $\Phi: \mathfrak{S}\left(\mathcal{H}_{A}\right) \rightarrow \mathfrak{S}\left(\mathcal{H}_{B}\right)$ with respect to a family $\mathfrak{S}$ of states in $\mathfrak{S}\left(\mathcal{H}_{A}\right)$ means existence of a quantum channel $\Psi: \mathfrak{S}\left(\mathcal{H}_{B}\right) \rightarrow \mathfrak{S}\left(\mathcal{H}_{A}\right)$ such that $\Psi(\Phi(\rho))=\rho$ for all $\rho \in \mathfrak{S}[18,23]$.

The notion of reversibility of a channel naturally arises in analysis of different general questions of quantum information theory, in particular, of conditions for preserving entropic characteristics of quantum states under action of a channel [10, 17, 18, 22, 23]. For instance, it follows from Petz's theorem (cf. [17, 23]) that the Holevo quantity 1 of an ensemble $\left\{\pi_{i}, \rho_{i}\right\}$ of quantum states is preserved under action of a quantum channel $\Phi$, i.e.

$$
\chi\left(\left\{\pi_{i}, \Phi\left(\rho_{i}\right)\right\}\right)=\chi\left(\left\{\pi_{i}, \rho_{i}\right\}\right)
$$

if and only if the channel $\Phi$ is reversible with respect to the family $\left\{\rho_{i}\right\}$.

A general criterion for reversibility of a quantum channel expressed in terms of von Neumann algebras theory is obtained in [17] (see also [18]). By using this criterion and the notion of complementary channel conditions for reversibility of a quantum channel with respect to complete families of states, in particular, of pure states are obtained in [25]. These conditions can be specified and reformulated for analysis of reversibility with respect to noncomplete families. Moreover, their "necessary" parts can be expressed in terms of weak complementary channel by using the "face property" of a set of all channels reversible with respect to a given family of states. These generalizations and their corollaries, in particular, several criteria for reversibility of a channel with respect to families of orthogonal mixed states are considered in Section III.

In Section IV we apply these conditions to Bosonic linear (quasi-free) channels. We show that a noisy Bosonic linear channel is reversible neither with respect to any complete family of pure states nor with respect to any complete family of orthogonal states containing a finite rank state (but it may be reversible with respect to complete family of orthogonal infinite rank states). Then we focus attention on analysis of reversibility of such channels with respect to noncomplete orthogonal and nonorthogonal families. The obtained conditions are reformulated for Bosonic Gaussian channels playing a central role in quantum information theory [3, 9, 12, 16].

The obtained results imply conditions for (non)-preserving the Holevo quantity of arbitrary (discrete or continuous) ensembles of states under action of a quantum channel. They are considered in Section V.

\footnotetext{
${ }^{1}$ The Holevo quantity (defined in Section II) provides an upper bound for accessible classical information which can be obtained by applying a quantum measurement [12, 21].
} 


\section{Preliminaries}

Let $\mathcal{H}$ be a separable Hilbert space, $\mathfrak{B}(\mathcal{H})$ and $\mathfrak{T}(\mathcal{H})$ - the Banach spaces of all bounded operators in $\mathcal{H}$ and of all trace-class operators in $\mathcal{H}$ correspondingly, $\mathfrak{S}(\mathcal{H})$ - the closed convex subset of $\mathfrak{T}(\mathcal{H})$ consisting of positive operators with unit trace called states [12, 21]. Denote by $\mathfrak{B}_{+}(\mathcal{H})$ the cone of positive operators in $\mathfrak{B}(\mathcal{H})$.

We will use the Greek letters $\rho, \sigma, \ldots$ for trace-class operators (not only for states) to distinguish their from bounded operators which will be denoted $A, B, \ldots$

The support supp $\rho$ of a positive operator $\rho$ is the orthogonal complement to its kernel.

Denote by $I_{\mathcal{H}}$ and $\operatorname{Id}_{\mathcal{H}}$ the unit operator in a Hilbert space $\mathcal{H}$ and the identity transformation of the Banach space $\mathfrak{T}(\mathcal{H})$ correspondingly.

Let $H(\rho)$ and $H(\rho \| \sigma)$ be respectively the von Neumann entropy of a state $\rho$ and the quantum relative entropy of states $\rho$ and $\sigma$ [12, 21].

A finite or countable collection of states $\left\{\rho_{i}\right\}$ with the corresponding probability distribution $\left\{\pi_{i}\right\}$ is called ensemble and denoted $\left\{\pi_{i}, \rho_{i}\right\}$. Its Holevo quantity is defined as follows

$$
\chi\left(\left\{\pi_{i}, \rho_{i}\right\}\right) \doteq \sum_{i} \pi_{i} H\left(\rho_{i} \| \bar{\rho}\right)=H(\bar{\rho})-\sum_{i} \pi_{i} H\left(\rho_{i}\right),
$$

where $\bar{\rho} \doteq \sum_{i} \pi_{i} \rho_{i}$ is the average state of this ensemble and the second formula is valid under the condition $H(\bar{\rho})<+\infty[12,21]$.

A completely positive trace preserving linear map $\Phi: \mathfrak{T}\left(\mathcal{H}_{A}\right) \rightarrow \mathfrak{T}\left(\mathcal{H}_{B}\right)$ is called quantum channel [12, 21]. It has the Kraus representation

$$
\Phi(\rho)=\sum_{k} V_{k} \rho V_{k}^{*}, \quad \rho \in \mathfrak{T}\left(\mathcal{H}_{A}\right)
$$

where $\left\{V_{k}\right\}$ is a set of linear operators from $\mathcal{H}_{A}$ into $\mathcal{H}_{B}$ such that $\sum_{k} V_{k}^{*} V_{k}=I_{\mathcal{H}_{A}}$.

We will use unitary dilation of a quantum channel [3, 4, 12]: for a channel $\Phi: \mathfrak{T}\left(\mathcal{H}_{A}\right) \rightarrow$ $\mathfrak{T}\left(\mathcal{H}_{B}\right)$ one can find separable Hilbert spaces $\mathcal{H}_{D}, \mathcal{H}_{E}$ for which $\mathcal{H}_{A} \otimes \mathcal{H}_{D} \subseteq \mathcal{H}_{B} \otimes \mathcal{H}_{E}=\mathcal{H}$, a state $\rho_{D}$ in $\mathfrak{S}\left(\mathcal{H}_{D}\right)$ and an unitary operator $U$ in the space $\mathcal{H}$ such that this channel can be represented as follows

$$
\Phi(\rho)=\operatorname{Tr}_{\mathcal{H}_{E}} U \rho \otimes \rho_{D} U^{*}, \quad \rho \in \mathfrak{T}\left(\mathcal{H}_{A}\right) .
$$

The quantum channel

$$
\widehat{\Phi}^{w}(\rho)=\operatorname{Tr}_{\mathcal{H}_{B}} U \rho \otimes \rho_{D} U^{*}, \quad \rho \in \mathfrak{T}\left(\mathcal{H}_{A}\right)
$$

is called weak complementary to the channel $\Phi$ [2], [12, Ch.6]. If the state $\rho_{D}$ is pure then (2) is the Stinespring representation of the channel $\Phi$ and the channel defined by (3) coincides with the complementary channel $\widehat{\Phi}$ to the channel $\Phi$ [7]. The weak complementary channel is not uniquely defined (it depends on representation (2)), but the complementary channel 
is unique: if $\widehat{\Phi}^{\prime}: \mathfrak{T}\left(\mathcal{H}_{A}\right) \rightarrow \mathfrak{T}\left(\mathcal{H}_{E^{\prime}}\right)$ is a channel defined by (3) via another representation (2) (with pure state $\rho_{D}^{\prime}$ ) then the channels $\widehat{\Phi}$ and $\widehat{\Phi}^{\prime}$ are isometrically equivalent in the sense of the following definition [15, the Appendix].

Definition 1. Channels $\Phi: \mathfrak{T}\left(\mathcal{H}_{A}\right) \rightarrow \mathfrak{T}\left(\mathcal{H}_{B}\right)$ and $\Phi^{\prime}: \mathfrak{T}\left(\mathcal{H}_{A}\right) \rightarrow \mathfrak{T}\left(\mathcal{H}_{B}^{\prime}\right)$ are isometrically equivalent if there exists a partial isometry $W: \mathcal{H}_{B} \rightarrow \mathcal{H}_{B^{\prime}}$ such that

$$
\Phi^{\prime}(\rho)=W \Phi(\rho) W^{*}, \quad \Phi(\rho)=W^{*} \Phi^{\prime}(\rho) W, \quad \rho \in \mathfrak{T}\left(\mathcal{H}_{A}\right) .
$$

The notion of isometrical equivalence is very close to the notion of unitary equivalence (see the remark after Definition 2 in [25]).

Definition 2. A channel $\Phi: \mathfrak{T}\left(\mathcal{H}_{A}\right) \rightarrow \mathfrak{T}\left(\mathcal{H}_{B}\right)$ is called classical-quantum of discrete type (briefly, discrete $c-q$ channel) if it has the following representation

$$
\Phi(\rho)=\sum_{i=1}^{\operatorname{dim} \mathcal{H}_{A}}\langle i|\rho| i\rangle \sigma_{i}, \quad \rho \in \mathfrak{T}\left(\mathcal{H}_{A}\right),
$$

where $\{|i\rangle\}$ is an orthonormal basis in $\mathcal{H}_{A}$ and $\left\{\sigma_{i}\right\}$ is a collection of states in $\mathfrak{S}\left(\mathcal{H}_{B}\right)$.

We use the term "discrete" in this definition, since in infinite dimensions there exist channels naturally called classical-quantum which have no representation (4), for example, Bosonic Gaussian c-q channels [12, Ch.12].

Discrete c-q channel (4), for which $\sigma_{i}=\sigma$ for all $i$, is a completely depolarizing channel: $\Phi(\rho)=\sigma \operatorname{Tr} \rho$.

Following [18, 22] introduce the basic notion of this paper.

Definition 3. A channel $\Phi: \mathfrak{T}\left(\mathcal{H}_{A}\right) \rightarrow \mathfrak{T}\left(\mathcal{H}_{B}\right)$ is reversible with respect to a family $\mathfrak{S} \subseteq \mathfrak{S}\left(\mathcal{H}_{A}\right)$ if there exists a channel $\Psi: \mathfrak{T}\left(\mathcal{H}_{B}\right) \rightarrow \mathfrak{T}\left(\mathcal{H}_{A}\right)$ such that $\rho=\Psi \circ \Phi(\rho)$ for all $\rho \in \mathfrak{S}$.

In [17, 23] this property is called sufficiency of the channel $\Phi$ for the family $\mathfrak{S}$.

We will call $\Psi$ and $\mathfrak{S}$ in Def. 3 reversing channel and reversed family respectively.

Definition 4. A family $\mathfrak{S}$ of states in $\mathfrak{S}(\mathcal{H})$ is complete if for any nonzero positive operator $A$ in $\mathfrak{B}(\mathcal{H})$ there exists a state $\rho \in \mathfrak{S}$ such that $\operatorname{Tr} A \rho>0$.

A family $\left\{\left|\varphi_{\lambda}\right\rangle\left\langle\varphi_{\lambda}\right|\right\}_{\lambda \in \Lambda}$ of pure states in $\mathfrak{S}(\mathcal{H})$ is complete if and only if the linear hull of the family $\left\{\left|\varphi_{\lambda}\right\rangle\right\}_{\lambda \in \Lambda}$ is dense in $\mathcal{H}$.

By separability of $\mathcal{H}$ an arbitrary complete family of states in $\mathfrak{S}(\mathcal{H})$ contains a countable complete subfamily [17, Lemma 2].

\section{General conditions for reversibility}

Now we consider general conditions for reversibility of a channel with respect to arbitrary families of states (pure states for the most part). 
We begin with the following observation showing the "face property" of a set of all channels reversible with respect to a given family of states.

Proposition 1. Let $\Phi_{1}$ and $\Phi_{2}$ be quantum channels from $\mathfrak{T}\left(\mathcal{H}_{A}\right)$ to $\mathfrak{T}\left(\mathcal{H}_{B}\right)$ and $\Phi=$ $p \Phi_{1}+(1-p) \Phi_{2}$, where $p \in(0,1)$. If the channel $\Phi$ is reversible with respect to a family $\mathfrak{S}$ of states in $\mathfrak{S}\left(\mathcal{H}_{A}\right)$ then the channels $\Phi_{1}$ and $\Phi_{2}$ are reversible with respect to the family $\mathfrak{S}$.

Proof. By Definition 3 reversibility of a channel with respect to a given family of states is equivalent to its reversibility with respect to any dense countable subfamily of this family. So, since the space $\mathfrak{T}\left(\mathcal{H}_{A}\right)$ is separable, we may assume that the family $\mathfrak{S}$ is countable.

Let $\mathfrak{S}=\left\{\rho_{i}\right\}$ and $\left\{\pi_{i}\right\}$ be a nondegenerate probability distribution with finite Shannon entropy. Then the Holevo quantity of the ensemble $\left\{\pi_{i}, \rho_{i}\right\}$ is finite. Let $\bar{\rho}=\sum_{i} \pi_{i} \rho_{i}$ be the average state of this ensemble. By reversibility of the channel $\Phi$ with respect to the family $\mathfrak{S}$ we have

$$
\begin{aligned}
\sum_{i} \pi_{i} H\left(\rho_{i} \| \bar{\rho}\right) & =\sum_{i} \pi_{i} H\left(\Phi\left(\rho_{i}\right) \| \Phi(\bar{\rho})\right) \\
& =\sum_{i} \pi_{i} H\left(p \Phi_{1}\left(\rho_{i}\right)+(1-p) \Phi_{2}\left(\rho_{i}\right) \| p \Phi_{1}(\bar{\rho})+(1-p) \Phi_{2}(\bar{\rho})\right) \\
& \leq p \sum_{i} \pi_{i} H\left(\Phi_{1}\left(\rho_{i}\right) \| \Phi_{1}(\bar{\rho})\right)+(1-p) \sum_{i} \pi_{i} H\left(\Phi_{2}\left(\rho_{i}\right) \| \Phi_{2}(\bar{\rho})\right) \\
& \leq p \sum_{i} \pi_{i} H\left(\rho_{i} \| \bar{\rho}\right)+(1-p) \sum_{i} \pi_{i} H\left(\rho_{i} \| \bar{\rho}\right)=\sum_{i} \pi_{i} H\left(\rho_{i} \| \bar{\rho}\right)
\end{aligned}
$$

where the inequalities follow from monotonicity and joint convexity of the relative entropy. Thus equalities hold in both these inequalities and hence the channels $\Phi_{1}$ and $\Phi_{2}$ preserve the Holevo quantity of the ensemble $\left\{\pi_{i}, \rho_{i}\right\}$. By Theorem 2 in [17] (with the Remark after it) this implies reversibility the channels $\Phi_{1}$ and $\Phi_{2}$ with respect to the family $\mathfrak{S}$.

Note that the assertion of Proposition 1 is not inverted: reversibility of channels $\Phi_{1}$ and $\Phi_{2}$ with respect to some family $\mathfrak{S}$ does not imply reversibility of their convex mixture with respect to this family. The simplest example is given by unitary channels $\Phi_{1}$ and $\Phi_{2}$.

Petz's theorem implies the following necessary condition for reversibility of a quantum channel with respect to complete families of states (for families of orthogonal states this condition is also sufficient for reversibility).

Theorem 1. Let $\mathfrak{S}=\left\{\rho_{i}\right\}$ be a complete family of states in $\mathfrak{S}\left(\mathcal{H}_{A}\right),\left\{\pi_{i}\right\}$ a nondegenerate probability distribution and $\left\{A_{i}=\pi_{i}[\bar{\rho}]^{-1 / 2} \rho_{i}[\bar{\rho}]^{-1 / 2}\right\}$ - the corresponding resolution of the identity in $\mathcal{H}_{A}$, where $\bar{\rho}=\sum_{i} \pi_{i} \rho_{i}$.

If a channel $\Phi: \mathfrak{T}\left(\mathcal{H}_{A}\right) \rightarrow \mathfrak{T}\left(\mathcal{H}_{B}\right)$ is reversible with respect to the family $\mathfrak{S}$ then any its weak complementary channel $\widehat{\Phi}^{w}$ has the Kraus representation

$$
\widehat{\Phi}^{w}(\rho)=\sum_{i, j} W_{i j} \rho W_{i j}^{*} \quad \text { such that } \quad A_{i}=\sum_{j} W_{i j}^{*} W_{i j} \quad \text { for all } i \text {. }
$$


It follows, in particular, that

$$
\max _{i, j} \operatorname{rank} W_{i j} \leq \max _{i} \operatorname{rank} \rho_{i} \quad \text { and } \min _{i} \max _{j} \operatorname{rank} W_{i j} \leq \min _{i} \operatorname{rank} \rho_{i} .
$$

If $\operatorname{supp} \rho_{i} \perp \operatorname{supp} \rho_{k}$ for all $i \neq k$ then existence of Kraus representation (5) for the channel $\widehat{\Phi}^{w}=\widehat{\Phi}$ is equivalent to reversibility of the channel $\Phi$ with respect to the family $\mathfrak{S}$.

Proof. The Kraus representation (5) for the complementary channel $\widehat{\Phi}$ is constructed in the proof of Theorem 3 in [25].

Let $\widehat{\Phi}^{w}$ be a weak complementary channel to the channel $\Phi$ defined by formula (3) and $\rho_{D}=\sum_{k} \lambda_{k} \rho_{D}^{k}$ a pure states decomposition of the state $\rho_{D}$. Then $\Phi=\sum_{k} \lambda_{k} \Phi_{k}$ and $\widehat{\Phi}^{w}=\sum_{k} \lambda_{k} \widehat{\Phi}_{k}^{w}$, where $\Phi_{k}$ and $\widehat{\Phi}_{k}^{w}$ are channels defined by formulae (2) and (3) with $\rho_{D}^{k}$ instead of $\rho_{D}$. Since the state $\rho_{D}^{k}$ is pure, we have $\widehat{\Phi}_{k}^{w}=\widehat{\Phi}_{k}$ for each $k$.

By Proposition 1 reversibility of the channel $\Phi$ with respect to the family $\mathfrak{S}$ implies reversibility of all the channels $\Phi_{k}$ with respect to this family. Thus, as mentioned before, all the channels $\widehat{\Phi}_{k}^{w}=\widehat{\Phi}_{k}$ have Kraus representation (5). Hence the same property holds for the channel $\widehat{\Phi}^{w}=\sum_{k} \lambda_{k} \widehat{\Phi}_{k}^{w}$.

If $\operatorname{supp} \rho_{i} \perp \operatorname{supp} \rho_{k}$ for all $i \neq k$ then $A_{i}$ is the projector onto $\operatorname{supp} \rho_{i}$ for each $i$. Representation (5i) of the channel $\widehat{\Phi}$ implies $\widehat{\widehat{\Phi}}(\rho)=\sum_{i, j, k, l} \operatorname{Tr}\left[W_{i j} \rho W_{k l}^{*}\right]|i \otimes j\rangle\langle k \otimes l|$ (cf. [15]) and hence $\operatorname{supp} \widehat{\widehat{\Phi}}\left(\rho_{i}\right) \perp \operatorname{supp} \widehat{\widehat{\Phi}}\left(\rho_{k}\right)$ for all $i \neq k$. It follows, since $\Phi$ and $\widehat{\hat{\Phi}}$ are isometrically equivalent channels, that the channel $\Phi$ is reversible with respect to the family $\left\{\rho_{i}\right\}$ (by Lemma 1 in [25]).

In analysis of reversibility of a channel with respect to noncomplete families of pure states we will need the following notion.

Definition 5. The restriction of a channel $\Phi: \mathfrak{T}\left(\mathcal{H}_{A}\right) \rightarrow \mathfrak{T}\left(\mathcal{H}_{B}\right)$ to the subspace $\mathfrak{T}\left(\mathcal{H}_{0}\right)$ of $\mathfrak{T}\left(\mathcal{H}_{A}\right)$, where $\mathcal{H}_{0}$ is a nontrivial subspace of $\mathcal{H}_{A}$, is called subchannel of $\Phi$ corresponding to the subspace $\mathcal{H}_{0}$ and is denoted $\left.\Phi\right|_{\mathfrak{T}\left(\mathcal{H}_{0}\right)}$.

The (weak) complementary channel to the subchannel of a channel $\Phi$ corresponding to any subspace $\mathcal{H}_{0} \subset \mathcal{H}_{A}$ coincides with the subchannel of the (weak) complementary channel $\widehat{\Phi}$ corresponding to the subspace $\mathcal{H}_{0}$, i.e.

$$
\widehat{\Psi}=\left.\widehat{\Phi}\right|_{\mathfrak{T}\left(\mathcal{H}_{0}\right)} \quad \text { and } \quad \widehat{\Psi}^{w}=\left.\widehat{\Phi}^{w}\right|_{\mathfrak{T}\left(\mathcal{H}_{0}\right)}, \quad \text { where } \quad \Psi=\left.\Phi\right|_{\mathfrak{T}\left(\mathcal{H}_{0}\right)} .
$$

Remark 1. It follows from (77) that the reversibility conditions in Theorem 11 are generalized to noncomplete family $\mathfrak{S}$ by replacing the channels $\widehat{\Phi}^{w}$ and $\widehat{\Phi}$ by their subchannels corresponding to the subspace $\mathcal{H}_{A}^{\mathfrak{S}}=\bigvee_{\rho \in \mathfrak{S}} \operatorname{supp} \rho$.

Remark 2. The first relation in (6) shows that reversibility of a channel $\Phi$ with respect to a complete family of states of rank $\leq r$ implies that any its weak complementary channel $\widehat{\Phi}^{w}$ is $r$-partially entanglement-breaking [5]. Thus, by Theorem 1 and Remark [1, to prove that a channel $\Phi$ is not reversible with respect to any family $\mathfrak{S}$ of states of rank $\leq r$ it 
suffices to find its weak complementary channel $\widehat{\Phi}^{w}$ and a state $\omega$ in $\mathfrak{S}\left(\mathcal{H}_{A}^{\mathfrak{S}} \otimes \mathcal{K}\right)$ such that

$$
\text { either } S N\left(\widehat{\Phi}^{w} \otimes \operatorname{Id}_{\mathcal{K}}(\omega)\right)>r \quad \text { or } \quad E\left(\widehat{\Phi}^{w} \otimes \operatorname{Id}_{\mathcal{K}}(\omega)\right)>\log r
$$

where $S N$ is the Schmidt number and $E$ is any convex entanglement monotone coinciding on the set of pure states with the entropy of a partial state, in particular, $E=E o F$ [24].

The second relation in (6) can be used to show nonreversibility of a channel $\Phi$ with respect to families containing at least one finite rank state. In particular, for complete families it suffices to find a weak complementary channel $\widehat{\Phi}^{w}$ such that any its Kraus representation (1) consists of infinite rank operators $V_{k}$. We will use this way in the proof of Corollary 5 in Section IV.2.

Theorem 1 gives the following criteria for reversibility with respect to orthogonal families.

Corollary 1. Let $\Phi: \mathfrak{T}\left(\mathcal{H}_{A}\right) \rightarrow \mathfrak{T}\left(\mathcal{H}_{B}\right)$ be a quantum channel and $\mathfrak{S}=\left\{\rho_{i}\right\}$ a family of mutually orthogonal states in $\mathfrak{S}\left(\mathcal{H}_{A}\right)$. Let $P_{i}$ be the projector on the support of the state $\rho_{i}$ for each $i$ and $\mathcal{H}_{A}^{\mathfrak{S}}=\bigoplus_{i} \operatorname{supp} \rho_{i}$. The following statements are equivalent:

(i) the channel $\Phi$ is reversible with respect to the family $\mathfrak{S}$;

(ii) the subchannel $\left.\Phi\right|_{\mathfrak{T}\left(\mathcal{H} \mathcal{S}_{A}^{\mathfrak{S}}\right)}$ is isometrically equivalent to the channel

$$
\mathfrak{T}\left(\mathcal{H}_{A}^{\mathfrak{S}}\right) \ni \rho \mapsto \Psi(\rho)=\sum_{i, j, k, l} \operatorname{Tr}\left[W_{i j} \rho W_{k l}^{*}\right]|i \otimes j\rangle\langle k \otimes l|
$$

where $\left\{W_{i j}\right\}$ is a set of operators such that $\sum_{j} W_{i j}^{*} W_{i j}=P_{i}$ for all $i$;

(iii) $\widehat{\Phi}(\rho)=\sum_{i, j} W_{i j} \rho W_{i j}^{*}$ for all $\rho \in \mathfrak{T}\left(\mathcal{H}_{A}^{\mathfrak{S}}\right)$, where $\left\{W_{i j}\right\}$ is the same set as in (ii);

(iv) $P_{i} \widehat{\Phi}^{*}(A) P_{k}=0$ for all $A \in \mathfrak{B}\left(\mathcal{H}_{E}\right)$ and all $i \neq k$;

(v) $\left\{P_{i}\right\} \subset P \Phi^{*}\left(\mathfrak{B}_{+}\left(\mathcal{H}_{B}\right)\right) P$, where $P=\sum_{i} P_{i}$ is the projector onto $\mathcal{H}_{A}^{\mathfrak{S}}$.

If (i) holds then (iii) and (iv) are valid for any weak complementary channel $\widehat{\Phi}^{w}$ to the channel $\Phi$.

If the family $\mathfrak{S}$ is complete (i.e. $\mathcal{H}_{A}^{\mathfrak{S}}=\mathcal{H}_{A}$ ) then (ii) gives a description (up to isometrical equivalence) of the set of all quantum channels reversible with respect to $\mathfrak{S}$.

Proof. By passing to the subchannel of $\Phi$ corresponding to the subspace $\mathcal{H}_{A}^{\mathfrak{S}}$ we may consider that $\mathfrak{S}$ is a complete family.

(i) $\Leftrightarrow$ (iii) follows from Theorem 1 and Remark 1, (ii) $\Leftrightarrow$ (iii) follows from the standard representation of a complementary channel [15, formula (11)]. (iii) $\Rightarrow$ (iv) is easily verified.

(iv) $\Rightarrow$ (i) For given $i$ and $k \neq i$ it follows from (iv) that $\widehat{\Phi}(|\varphi\rangle\langle\psi|)=0$ for any vectors $\varphi \in \operatorname{supp} \rho_{i}$ and $\psi \in \operatorname{supp} \rho_{k}$. By the definition of a complementary channel this implies $\operatorname{supp} \Phi(|\varphi\rangle\langle\varphi|) \perp \operatorname{supp} \Phi(|\psi\rangle\langle\psi|)$. It follows that $\operatorname{supp} \Phi\left(\rho_{i}\right) \perp \operatorname{supp} \Phi\left(\rho_{k}\right)$ for all $i \neq k$ and hence the channel $\Phi$ is reversible with respect to the family $\left\{\rho_{i}\right\}$. 
(ii) $\Rightarrow(\mathrm{v})$ Since $\Psi^{*}(A)=\sum_{i, j, k, l}\langle k \otimes l|A| i \otimes j\rangle W_{k l}^{*} W_{i j}$, we have $P_{i}=\sum_{j} W_{i j}^{*} W_{i j}=$ $\Psi^{*}\left(|i\rangle\langle i| \otimes I_{\mathcal{H}\{|j\rangle\}}\right)$, where $\mathcal{H}^{\{|j\rangle\}}$ is the Hilbert space with the basis $\{|j\rangle\}$.

$(\mathrm{v}) \Rightarrow$ (iii) follows from the proof of Theorem 3 in [25].

The last assertion of the corollary follows from Theorem 1 and Remark 1 .

Now we consider conditions for reversibility of a quantum channel with respect to arbitrary families of pure states.

By Lemma 5 in [25] any family $\mathfrak{S}$ of pure states in $\mathfrak{S}(\mathcal{H})$ has the unique (finite or countable) decomposition

$$
\mathfrak{S}=\bigcup_{k=1}^{n} \mathfrak{S}_{k} \quad\left(n \leq \operatorname{dim} \bigvee_{\rho \in \mathfrak{S}} \operatorname{supp} \rho\right)
$$

where $\left\{\mathfrak{S}_{k}\right\}_{k=1}^{n}$ is a collection of orthogonally non-decomposable (OND) families (this means that there is no subspace $\mathcal{H}_{0}$ such that some states (not all) from $\mathfrak{S}_{k}$ lie in $\mathcal{H}_{0}$, while the others - in $\left.\mathcal{H}_{0}^{\perp}\right)$ mutually orthogonal in the sense that $\rho \perp \sigma$ if $\rho \in \mathfrak{S}_{k}$ and $\sigma \in \mathfrak{S}_{l}, k \neq l$.

The following theorem is an extended and strengthened version of Theorem 4 in [25].

Theorem 2. Let $\Phi: \mathfrak{T}\left(\mathcal{H}_{A}\right) \rightarrow \mathfrak{T}\left(\mathcal{H}_{B}\right)$ be a quantum channel and $\mathfrak{S}$ a family of pure states in $\mathfrak{S}\left(\mathcal{H}_{A}\right)$ with decomposition (8) into OND subfamilies. Let $\mathcal{H}_{A}^{\mathfrak{S}}=\bigvee_{\rho \in \mathfrak{S}} \operatorname{supp} \rho, \mathcal{H}_{B}^{\mathfrak{S}}=$ $\bigvee_{\rho \in \mathfrak{S}} \operatorname{supp} \Phi(\rho)$

$$
m=\min \left\{\operatorname{dim}\left[\operatorname{ker} P_{\mathfrak{S}} \Phi^{*}(\cdot) P_{\mathfrak{S}} \cap \mathfrak{B}\left(\mathcal{H}_{B}^{\mathfrak{S}}\right)\right]+1, \operatorname{dim} \mathcal{H}_{B}^{\mathfrak{S}}\right\}
$$

where $P_{\mathfrak{S}}$ is the projector on the subspace $\mathcal{H}_{A}^{\mathfrak{S}}$, and $\left\{P_{k}\right\}_{k=1}^{n}$ the orthogonal resolution of the identity in $\mathcal{H}_{A}^{\mathfrak{S}}$ corresponding to decomposition (8). The following statements are equivalent:

(i) the channel $\Phi$ is reversible with respect to the family $\mathfrak{S}$;

(ii) the channel $\Phi$ is reversible with respect to the family

$$
\hat{\mathfrak{S}}=\left\{\rho \in \mathfrak{S}\left(\mathcal{H}_{A}^{\mathfrak{S}}\right) \mid \rho=\sum_{k=1}^{n} P_{k} \rho P_{k}\right\} ;
$$

(iii) the subchannel $\left.\widehat{\Phi}\right|_{\mathfrak{T}(\mathcal{H}} ^{\mathfrak{S})}$ is a discrete c-q channel having the representation

$$
\widehat{\Phi}(\rho)=\sum_{k=1}^{n}\left[\operatorname{Tr} P_{k} \rho\right] \sigma_{k}, \quad \rho \in \mathfrak{T}\left(\mathcal{H}_{A}^{\mathfrak{S}}\right),
$$

where $\left\{\sigma_{k}\right\}$ is a set of states in $\mathfrak{S}\left(\mathcal{H}_{E}\right)$ such that $\operatorname{rank} \sigma_{k} \leq m$ for all $k$;

(iv) the subchannel $\left.\Phi\right|_{\left.\mathfrak{T}_{(\mathcal{H}}^{\mathfrak{S}}\right)}$ is isometrically equivalent to the channel

$$
\Psi(\rho)=\sum_{k, l=1}^{n} P_{k} \rho P_{l} \otimes \sum_{p, t=1}^{m}\left\langle\psi_{t}^{l} \mid \psi_{p}^{k}\right\rangle|p\rangle\langle t|
$$

from $\mathfrak{T}\left(\mathcal{H}_{A}^{\mathfrak{S}}\right)$ into $\mathfrak{T}\left(\mathcal{H}_{A}^{\mathfrak{S}} \otimes \mathcal{H}_{m}\right)$, where $\left\{\left|\psi_{p}^{k}\right\rangle\right\}$ is a collection of vectors in a separable Hilbert space such that $\sum_{p=1}^{m}\left\|\psi_{p}^{k}\right\|^{2}=1$ and $\left\langle\psi_{t}^{k} \mid \psi_{p}^{k}\right\rangle=0$ for all $p \neq t$ for each $k$ and $\{|p\rangle\}_{p=1}^{m}$ is an orthonormal basis in $\mathcal{H}_{m}$. 
If $\widehat{\Phi}^{w}$ is a weak complementary channel to the channel $\Phi$ defined by (3) via the state $\rho_{D}$ then (i) implies that $\left.\widehat{\Phi}^{w}\right|_{\mathfrak{T}\left(\mathcal{H}_{A}^{\mathfrak{S}}\right)}$ is a discrete c-q channel having representation (9) in which $\left\{\sigma_{k}\right\}$ is a set of states in $\mathfrak{S}\left(\mathcal{H}_{E}\right)$ such that $\operatorname{rank} \sigma_{k} \leq \operatorname{dim} \mathcal{H}_{B}^{\mathfrak{S}} \times \operatorname{rank} \rho_{D}$ for all $k$.

Proof. The first assertion of the theorem follows from Theorem 4 in [25] applied to the subchannel of $\Phi$ corresponding to the subspace $\mathcal{H}_{A}^{\mathfrak{S}}$ and (7).

Let $\widehat{\Phi}^{w}$ be a weak complementary channel to the channel $\Phi$ defined by formula (3) and $\rho_{D}=\sum_{i=1}^{r} \lambda_{i} \rho_{D}^{i}$ be a pure states decomposition of the state $\rho_{D}$, where $r=\operatorname{rank} \rho_{D}$. Then $\Phi=\sum_{i=1}^{r} \lambda_{i} \Phi_{i}$ and $\widehat{\Phi}^{w}=\sum_{i=1}^{r} \lambda_{i} \widehat{\Phi}_{i}^{w}$, where $\Phi_{i}$ and $\widehat{\Phi}_{i}^{w}$ are channels defined by formulae (2) and (3) with $\rho_{D}^{i}$ instead of $\rho_{D}$. Since the state $\rho_{D}^{i}$ is pure, we have $\widehat{\Phi}_{i}^{w}=\widehat{\Phi}_{i}$ for each $i$.

By Proposition 1 reversibility of the channel $\Phi$ with respect to the family $\mathfrak{S}$ implies reversibility of the channels $\Phi_{i}, i=\overline{1, r}$, with respect to this family. By the first assertion of the theorem $\widehat{\Phi}_{i}^{w}(\rho)=\widehat{\Phi}_{i}(\rho)=\sum_{k=1}^{n}\left[\operatorname{Tr} P_{k} \rho\right] \sigma_{k}^{i}$ for all $\rho \in \mathfrak{S}\left(\mathcal{H}_{A}^{\mathfrak{S}}\right)$, where $\left\{\sigma_{k}^{i}\right\}$ is a set of states in $\mathfrak{S}\left(\mathcal{H}_{E}\right)$ such that $\operatorname{rank} \sigma_{k}^{i} \leq \operatorname{dim} \mathcal{H}_{B}^{\mathfrak{S}}$, for each $i$. Hence

$$
\widehat{\Phi}^{w}(\rho)=\sum_{i=1}^{r} \lambda_{i} \widehat{\Phi}_{i}^{w}(\rho)=\sum_{k=1}^{n}\left[\operatorname{Tr} P_{k} \rho\right] \sum_{i=1}^{r} \lambda_{i} \sigma_{k}^{i}, \quad \rho \in \mathfrak{T}\left(\mathcal{H}_{A}^{\mathfrak{S}}\right) .
$$

Remark 3. If the family $\mathfrak{S}$ in Theorem 2 is nonorthogonal then the collection $\left\{P_{k}\right\}_{k=1}^{n}$ contains at least one projector $P_{k_{0}}$ of rank $>1$. By the implication (i) $\Rightarrow$ (ii) in Theorem 2 reversibility of the channel $\Phi$ with respect to this family $\mathfrak{S}$ implies its reversibility with respect to the family of all states supported by the subspace $\mathcal{H}_{k_{0}}=P_{k_{0}}\left(\mathcal{H}_{A}\right)$, i.e. its perfect reversibility on the subspace $\mathcal{H}_{k_{0}}$ in terms of [12, Ch.10]. Theorem 2 also shows that reversibility of the channel $\Phi$ with respect to the family $\mathfrak{S}$ implies that the subchannels $\left.\widehat{\Phi}\right|_{\mathfrak{T}\left(\mathcal{H}_{k_{0}}\right)}$ and $\left.\widehat{\Phi}^{w}\right|_{\mathfrak{T}\left(\mathcal{H}_{k_{0}}\right)}$ are completely depolarizing.

Theorem 2 (with Remark 3) shows that analysis of reversibility properties of a quantum channel requires conditions for existence of discrete c-q subchannels and of completely depolarizing subchannels of the (weak) complementary channel. The following lemma gives such conditions expressed in terms of the kernel (null set) of a channel.

Lemma 1. Let $\Psi: \mathfrak{T}\left(\mathcal{H}_{A}\right) \rightarrow \mathfrak{T}\left(\mathcal{H}_{B}\right)$ be a quantum channel.

A) The channel $\Psi$ has no discrete $c-q$ subchannels if and only if the set ker $\Psi$ does not contain 1-rank operators.

B) The channel $\Psi$ has discrete $c-q$ subchannels but it has no completely depolarizing subchannels if and only if the set ker $\Psi$ contains 1-rank operators but it does not contain the operators

$$
|\varphi\rangle\langle\psi| \text { and }|\varphi\rangle\langle\varphi|-| \psi\rangle\langle\psi|
$$

simultaneously for all unit vectors $\varphi$ and $\psi$ in $\mathcal{H}_{A} .2$

\footnotetext{
${ }^{2}$ Since $\Psi$ is a trace-preserving map, any 1 -rank operator in $\operatorname{ker} \Psi$ has the form $|\varphi\rangle\langle\psi|$, where $\varphi$ and $\psi$ are orthogonal vectors in $\mathcal{H}_{A}$.
} 
C) The channel $\Psi$ has completely depolarizing subchannels if and only if the set $\operatorname{ker} \Psi$ contains operators (10) for some unit vectors $\varphi$ and $\psi$ in $\mathcal{H}_{A}$.

Proof. The assertions of the lemma follow from Lemma 3 in Appendix A.

To describe reversibility properties of a channel $\Phi$ with respect to families of pure states it is convenient to introduce the reversibility index $\operatorname{ri}(\Phi)=\left[\mathrm{ri}_{1}(\Phi), \mathrm{ri}_{2}(\Phi)\right]$, in which the both components take the values $0,1,2$. The first component $\mathrm{ri}_{1}(\Phi)$ characterizes reversibility of the channel $\Phi$ with respect to (w.r.t.) complete families of pure states as follows

$\operatorname{ri}_{1}(\Phi)=0$ if $\Phi$ is not reversible w.r.t. any complete family $\mathfrak{S}$ of pure states;

$\operatorname{ri}_{1}(\Phi)=1$ if $\Phi$ is reversible w.r.t. a complete orthogonal family $\mathfrak{S}$ of pure states but it is not reversible w.r.t. any complete nonorthogonal family $\mathfrak{S}$ of pure states;

$\operatorname{ri}_{1}(\Phi)=2$ if $\Phi$ is reversible w.r.t. a complete nonorthogonal family $\mathfrak{S}$ of pure states.

The value of $\operatorname{ri}_{1}(\Phi)$ can be interpreted geometrically as follows: $\mathrm{ri}_{1}(\Phi)>0$ means existence of an orthonormal basis $\left\{\left|\varphi_{i}\right\rangle\right\}$ of the space $\mathcal{H}_{A}$ such that

$$
\operatorname{supp} \Phi\left(\left|\varphi_{i}\right\rangle\left\langle\varphi_{i}\right|\right) \perp \operatorname{supp} \Phi\left(\left|\varphi_{j}\right\rangle\left\langle\varphi_{j}\right|\right) \quad \forall i \neq j
$$

if the channel $\Phi$ is perfectly reversible on a subspace spanned by some vectors of this basis then $\operatorname{ri}_{1}(\Phi)=2$, otherwise $\operatorname{ri}_{1}(\Phi)=1$ (this follows from Remark (3).

The second component $\mathrm{ri}_{2}(\Phi)$ characterizes reversibility of the channel $\Phi$ with respect to noncomplete families of pure states and is defined similarly to $\mathrm{ri}_{1}(\Phi)$ with the "complete family $\mathfrak{S}$ " replaced by "noncomplete family $\mathfrak{S}$ ".

So that $\operatorname{ri}(\Phi)=01$ means that the channel $\Phi$ is not reversible with respect to any family of pure states which is either complete or nonorthogonal, but it is reversible with respect to some noncomplete orthogonal family.

By Remark 3 the value of $\operatorname{ri}_{2}(\Phi)$ has the clear geometrical interpretation: $\operatorname{ri}_{2}(\Phi)=2$ means existence of a subspace of $\mathcal{H}_{A}$ on which the channel $\Phi$ is perfectly reversible, if there are no such subspaces but there exists an orthonormal set $\left\{\left|\varphi_{i}\right\rangle\right\}$ of vectors in $\mathcal{H}_{A}$ such that (11) holds then $\operatorname{ri}_{2}(\Phi)=1$. This implies the following observation.

Remark 4. If $\Phi$ is a finite dimensional channel then $\operatorname{ri}_{2}(\Phi)$ characterizes positivity of one-shot zero-error capacities of $\Phi$ as follows:

$$
\operatorname{ri}_{2}(\Phi)=0 \Leftrightarrow \bar{C}_{0}(\Phi)=0, \quad \operatorname{ri}_{2}(\Phi)=2 \Leftrightarrow \bar{Q}_{0}(\Phi)>0,
$$

(so, $\operatorname{ri}_{2}(\Phi)=1$ means that $\bar{C}_{0}(\Phi)>0$ but $\bar{Q}_{0}(\Phi)=0$ ), where $\bar{C}_{0}(\Phi)$ and $\bar{Q}_{0}(\Phi)$ are the one-shot zero-error classical and quantum capacities of the channel $\Phi$ respectively [20, 8].

It follows from the definition that the reversibility index can take the values

$00, \quad 01, \quad 02, \quad 11, \quad 12, \quad 22$. 
By using the below Corollary 2 it is easy to construct a channel with any reversibility index from the above list excepting the index 12. Existence of a channel $\Phi$ with $\operatorname{ri}(\Phi)=12$ is an interesting open question 3

Corollary 2. Let $\Phi$ be a quantum channel and $\widehat{\Phi}$ its complementary channel. Then

$$
\begin{aligned}
& \{\operatorname{ri}(\Phi)=00\} \Leftrightarrow\{\widehat{\Phi} \text { satisfies condition A of Lemma } 1\}, \\
& \{\operatorname{ri}(\Phi)=01\} \Leftrightarrow\{\widehat{\Phi} \text { is not discrete c- } q \text { and satisfies condition B of Lemma } 1\}, \\
& \{\operatorname{ri}(\Phi)=02\} \Leftrightarrow\{\widehat{\Phi} \text { is not discrete c- } q \text { and satisfies condition } C \text { of Lemma } 1]\}, \\
& \{\operatorname{ri}(\Phi)=11\} \Leftrightarrow\{\widehat{\Phi} \text { is discrete } C \text { - } Q \text { and satisfies condition } B \text { of Lemma } 1\}, \\
& \{\operatorname{ri}(\Phi)=12\} \Leftrightarrow\{\widehat{\Phi} \text { is discrete } C-Q \text { and satisfies condition } C \text { of Lemma } 1\}, \\
& \{\operatorname{ri}(\Phi)=22\} \Leftrightarrow\left\{\widehat{\Phi} \text { is discrete } c \text { - } q \text { channel (4) with } \sigma_{i}=\sigma_{j} \text { for some } i \neq j\right\},
\end{aligned}
$$

where "discrete C- $Q$ " denotes discrete $c$-q channel (4) with $\sigma_{i} \neq \sigma_{j}$ for all $i \neq j$.

For a weak complementary channel $\widehat{\Phi}^{w}$ to the channel $\Phi$ the following implications hold 4

$$
\begin{aligned}
& \{\operatorname{ri}(\Phi)=00\} \Leftarrow\left\{\widehat{\Phi}^{w} \text { satisfies condition A of Lemma } 1\right\} \\
& \{\operatorname{ri}(\Phi) \geq 02\} \Rightarrow\left\{\widehat{\Phi}^{w} \text { satisfies condition C of Lemma } 1\right\} \\
& \{\operatorname{ri}(\Phi) \geq 11\} \Rightarrow\left\{\widehat{\Phi}^{w} \text { is a discrete c- } q \text { channel }\right\} .
\end{aligned}
$$

Proof. All the above assertions follow from Theorem 2, Remark 3 and Lemma 1 .

We will show in the next section that the reversibility index takes the values 00,01,02 and 22 on the class of Bosonic linear channels.

\section{Reversibility of Bosonic linear channels}

Let $\mathcal{H}_{X}(X=A, B, \ldots)$ be the space of irreducible representation of the Canonical Commutation Relations (CCR)

$$
W_{X}(z) W_{X}\left(z^{\prime}\right)=\exp \left(-\frac{\mathrm{i}}{2} \Delta_{X}\left(z, z^{\prime}\right)\right) W_{X}\left(z^{\prime}+z\right), \quad z, z^{\prime} \in Z_{X},
$$

where $\left(Z_{X}, \Delta_{X}\right)$ is a symplectic space and $W_{X}(z)$ are the Weyl operators [3, 9], [12, Ch.12]. We will also use the symbol $\Delta_{X}$ for the matrix of the form $\Delta_{X}$, i.e. $\Delta_{X}\left(z, z^{\prime}\right)=z^{\top} \Delta_{X} z^{\prime}$. Denote by $s_{X}$ the number of modes of the system $X$, i.e. $2 s_{X}=\operatorname{dim} Z_{X} 5$

A Bosonic linear channel $\Phi_{K, f}: \mathfrak{T}\left(\mathcal{H}_{A}\right) \rightarrow \mathfrak{T}\left(\mathcal{H}_{B}\right)$ is defined via the action of its dual $\Phi_{K, f}^{*}: \mathfrak{B}\left(\mathcal{H}_{B}\right) \rightarrow \mathfrak{B}\left(\mathcal{H}_{A}\right)$ on the Weyl operators:

$$
\Phi_{K, f}^{*}\left(W_{B}(z)\right)=W_{A}(K z) f(z), \quad z \in Z_{B},
$$

\footnotetext{
${ }^{3}$ It seems intuitively that any discrete c-q channel (44) with $\sigma_{i} \neq \sigma_{j}$ for all $i \neq j$ can not have completely depolarizing subchannels, but I can not find a formal proof. I would be grateful for any comments.

${ }^{4}$ Here and in what follows $X_{1} X_{2} \leq Y_{1} Y_{2}$ means that $X_{1} \leq Y_{1}$ and $X_{2} \leq Y_{2}$.

${ }^{5}$ Some basic notions concerning symplectic spaces are presented in Appendix B.
} 
where $K: Z_{B} \rightarrow Z_{A}$ is a linear operator, and $f(z)$ is a complex continuous function on $Z_{B}$ such that $f(0)=1$ and the matrix with the elements $f\left(z_{s}-z_{r}\right) \exp \left(\frac{\mathrm{i}}{2} z_{s}^{\top}\left[\Delta_{B}-K^{\top} \Delta_{A} K\right] z_{r}\right)$ is positive for any finite subset $\left\{z_{s}\right\}$ of $Z_{B}$ [11, 13]. This channel is also called quasi-free [6].

We will assume existence of a Bosonic unitary dilation for the channel $\Phi_{K, f}$, i.e. existence of such Bosonic systems $D$ and $E$ that this channel can be represented as a restriction of a corresponding unitary evolution of the composite system $A D=B E$ (described by the symplectic space $Z=Z_{A} \oplus Z_{D}=Z_{B} \oplus Z_{E}$ ) provided that the system $D$ is in a particular state $\rho_{D}$. This means that

$$
\Phi_{K, f}^{*}\left(W_{B}(z)\right)=\operatorname{Tr}_{\mathcal{H}_{D}}\left(I_{\mathcal{H}_{A}} \otimes \rho_{D}\right) U_{T}^{*}\left(W_{B}(z) \otimes I_{\mathcal{H}_{E}}\right) U_{T}, \quad z \in Z_{B},
$$

where $U_{T}$ is the unitary operator in the space $\mathcal{H}_{A} \otimes \mathcal{H}_{D} \cong \mathcal{H}_{B} \otimes \mathcal{H}_{E}$ implementing the symplectic transformation

$$
T=\left[\begin{array}{ll}
K & L \\
K_{D} & L_{D}
\end{array}\right]
$$

of the space $Z$ (here $L: Z_{E} \rightarrow Z_{A}, K_{D}: Z_{B} \rightarrow Z_{D}, L_{D}: Z_{E} \rightarrow Z_{D}$ are appropriate linear operators) [3, 4, 12, 13]. Note that

$$
f(z)=\phi_{\rho_{D}}\left(K_{D} z\right)
$$

where $\phi_{\rho_{D}}$ is the characteristic function of the state $\rho_{D}$.

The weak complementary channel (see Section II) is defined as follows

$$
\begin{aligned}
{\left[\widehat{\Phi}_{K, f}^{w}\right]^{*}\left(W_{E}(z)\right) } & =\operatorname{Tr}_{\mathcal{H}_{D}}\left(I_{\mathcal{H}_{A}} \otimes \rho_{D}\right) U_{T}^{*}\left(I_{\mathcal{H}_{B}} \otimes W_{E}(z)\right) U_{T} \\
& =\operatorname{Tr}_{\mathcal{H}_{D}}\left(I_{\mathcal{H}_{A}} \otimes \rho_{D}\right)\left(W_{A}(L z) \otimes W_{D}\left(L_{D} z\right)\right) \\
& =W_{A}(L z) \phi_{\rho_{D}}\left(L_{D} z\right), \quad z \in Z_{E} .
\end{aligned}
$$

Thus $\widehat{\Phi}_{K, f}^{w}$ is a Bosonic linear channel as well. If the state $\rho_{D}$ is pure then $\widehat{\Phi}_{K, f}^{w}=\widehat{\Phi}_{K, f}$ is the complementary channel to the channel $\Phi_{K, f}$.

Remark 5. Unitary dilation (13)-(14) does not exist for all Bosonic linear channels (it suffices to note that (15) implies $|f(z)|=1 \Leftrightarrow f(z)=1$ ), but one can conjecture that any Bosonic linear channel can be transformed by appropriate displacement unitaries to Bosonic linear channel for which such dilation exists 6 This conjecture is true for Bosonic Gaussian channels (see Section IV.2).

\section{IV.1 Reversibility conditions}

We begin with the following observation concerning reversibility with respect to complete families.

\footnotetext{
${ }^{6}$ I would be grateful for any comments concerning this question.
} 
Proposition 2. Let $\Phi_{K, f}$ be a noisy (not noiseless [27]) Bosonic linear channel for which unitary dilation (13)-(14) exists. The channel $\Phi_{K, f}$ is not reversible with respect to a complete family $\mathfrak{S}$ in the following cases:

- $\mathfrak{S}$ consists of pure states;

- $\mathfrak{S}$ consists of orthogonal states at least one of which has finite rank.

The channel $\Phi_{K, f}$ is reversible with respect to a particular complete family $\mathfrak{S}$ of orthogonal infinite rank states if and only if $Z_{f} \doteq\left\{z \in Z_{B} \mid f(z)=1\right\} \neq\{0\}$.

Proof. It suffices to prove the first assertion in the second case, since reversibility of a channel with respect to a complete family of pure states implies its reversibility with respect to some complete family of orthogonal pure states [25, Corollary 2].

Let $\mathfrak{S}=\left\{\rho_{i}\right\}$ be a complete family of orthogonal states and $\left\{P_{i}\right\}$ the corresponding orthogonal resolution of the identity in $\mathcal{H}_{A}\left(P_{i}\right.$ is the projector onto supp $\left.\rho_{i}\right)$. Let $\Phi_{L, g}=\widehat{\Phi}_{K, f}^{w}$ be a weak complementary channel to the channel $\Phi$ defined by (16), where $g(z)=\phi_{\rho_{D}}\left(L_{D} z\right)$. Since $\Phi_{K, f}$ is not noiseless, $\Phi_{L, g}$ is not completely depolarizing, i.e. $L \neq 0$. By Corollary 1 $((\mathrm{i}) \Rightarrow(\mathrm{iv}))$ reversibility of the channel $\Phi_{K, f}$ with respect to the family $\mathfrak{S}$ implies

$$
g(z) W_{A}(L z)=g(z) \sum_{i} P_{i} W_{A}(L z) P_{i} \quad \forall z \in Z_{E} .
$$

If $\operatorname{rank} \rho_{i_{0}}<+\infty$ for some $i_{0}$ then (17) implies that the operator $W_{A}\left(z_{0}\right)$, where $z_{0}$ is a nonzero vector in $\operatorname{Ran} L$, commutes with the finite dimensional projector $P_{i_{0}}$. This contradicts to the well known fact that the Weyl operators have no purely point spectrum.

If $Z_{f}=\{0\}$ then, since (15) implies $Z_{f}=\operatorname{ker} K_{D}$, Lemma 2 below shows that $\operatorname{Ran} L=$ $\left[K\left(Z_{f}\right)\right]^{\perp}=Z_{A}$. It follows that the family $\left\{W_{A}(L z)\right\}_{z \in Z_{E}}$ acts irreducibly on $\mathcal{H}_{A}$ and hence (17) can not be valid for any orthogonal resolution of the identity $\left\{P_{i}\right\}$.

Let $Z_{f}=\operatorname{ker} K_{D} \neq\{0\}$. Consider the von Neumann algebras $\mathcal{A}$ and $\mathcal{B}$ generated respectively by the families $\left\{W_{A}(K z)\right\}_{z \in Z_{f}}$ and $\left\{W_{B}(z)\right\}_{z \in Z_{f}}$. By the second assertion of Lemma 2 below the restriction of the operator $K$ to the subspace $Z_{f}$ is nondegenerate and symplectic (i.e. $\Delta_{A}\left(K z_{1}, K z_{2}\right)=\Delta_{B}\left(z_{1}, z_{2}\right)$ for all $z_{1}, z_{2} \in Z_{f}$ ). This implies that the restriction of the dual map $\Phi_{K, f}^{*}$ to the algebra $\mathcal{B} \subseteq \mathfrak{B}\left(\mathcal{H}_{B}\right)$ is a $*$-isomorphism between the algebras $\mathcal{B}$ and $\mathcal{A}$ (see details in [26, Sect.2]). It follows that for any orthogonal resolution of the identity $\left\{P_{i}\right\}$ in $\mathcal{A}$ there exists an unique orthogonal resolution of the identity $\left\{Q_{i}\right\}$ in $\mathcal{B}$ such that $P_{i}=\Phi_{K, f}^{*}\left(Q_{i}\right)$ for all $i$ and hence

$$
\Phi_{K, f}\left(\mathfrak{S}\left(\mathcal{H}_{A}^{i}\right)\right) \subseteq \mathfrak{S}\left(\mathcal{H}_{B}^{i}\right) \quad \forall i
$$

where $\mathcal{H}_{A}^{i}=P_{i}\left(\mathcal{H}_{A}\right)$ and $\mathcal{H}_{B}^{i}=Q_{i}\left(\mathcal{H}_{B}\right)$ (so that $\mathcal{H}_{A}=\bigoplus_{i} \mathcal{H}_{A}^{i}$ and $\mathcal{H}_{B}=\bigoplus_{i} \mathcal{H}_{B}^{i}$ ).

Let $\left\{\rho_{i}\right\}$ be a family of states in $\mathfrak{S}\left(\mathcal{H}_{A}\right)$ such that $\operatorname{supp} \rho_{i}=\mathcal{H}_{A}^{i}$ for all $i$. It follows from (18) that the channel $\Phi_{K, f}$ is reversible with respect to the orthogonal family $\left\{\rho_{i}\right\}$ and that 
the simplest reversing channel has the form

$$
\Psi(\sigma)=\sum_{i}\left[\operatorname{Tr} Q_{i} \sigma\right] \rho_{i}, \quad \sigma \in \mathfrak{S}\left(\mathcal{H}_{B}\right)
$$

Now we consider reversibility of Bosonic linear channels with respect to arbitrary families of pure states. For these channels the reversibility index (introduced in Section III) can take the values $00,01,02,22$.

Theorem 3. Let $\Phi_{K, f}$ be a Bosonic linear channel for which unitary dilation (13)-(14) exists and $Z_{f} \doteq\left\{z \in Z_{B} \mid f(z)=1\right\}$. Then $\operatorname{ri}\left(\Phi_{K, f}\right)=22$ if and only if $\Phi_{K, f}$ is a noiseless channel (see [27]). Otherwise

$$
\begin{aligned}
& \left\{\operatorname{ri}\left(\Phi_{K, f}\right)=00\right\} \Leftrightarrow\left\{Z_{f}=\{0\}\right\}, \\
& \left\{\operatorname{ri}\left(\Phi_{K, f}\right)=01\right\} \Leftrightarrow\left\{Z_{f} \text { is a nontrivial isotropic subspace of } Z_{B}\right\}, \\
& \left\{\operatorname{ri}\left(\Phi_{K, f}\right)=02\right\} \Leftrightarrow\left\{\exists z_{1}, z_{2} \in Z_{f} \text { such that } \Delta_{B}\left(z_{1}, z_{2}\right) \neq 0\right\} .
\end{aligned}
$$

A description (in the Schrodinger representation) of reversed families of pure states for the channel $\Phi_{K, f}$ in the cases $\operatorname{ri}\left(\Phi_{K, f}\right)=01,02$ is given in Section IV.3.

Proof. The first assertion of the theorem follows from Proposition 2 .

In proving the second one we may consider (by using a purification procedure if necessary) that (13)-(14) is a Stinespring dilation for the channel $\Phi_{K, f}$, i.e. the state $\rho_{D}$ is pure. Then the complementary channel to the channel $\Phi_{K, f}$ is a Bosonic linear channel defined by (16).

Since (15) implies $Z_{f}=\operatorname{ker} K_{D}$, Lemma 2 below shows that $\operatorname{Ran} L=\left[K\left(Z_{f}\right)\right]^{\perp}$, ker $K \cap$ $Z_{f}=\{0\}$ and that $\Delta_{A}\left(K z_{1}, K z_{2}\right)=\Delta_{B}\left(z_{1}, z_{2}\right)$ for all $z_{1}, z_{2} \in Z_{f}$. Hence all the statements in (20) follow from Corollary 2 and Proposition 3 in Appendix A.

Remark 6. Sufficiency of the reversibility conditions (20) can be shown without using Corollary 2 by explicit construction of reversing channels for particular orthogonal and nonorthogonal families of pure states.

Reversibility of the channel $\Phi_{K, f}$ with respect to some orthogonal families of pure states under the condition $Z_{f} \neq\{0\}$ can be shown by repeating the arguments from the proof of Proposition 2 and by taking the family $\left\{\rho_{i}\right\}$ consisting of pure states such that $\operatorname{supp} \rho_{i} \subseteq \mathcal{H}_{A}^{i}$ for all $i$. The simplest reversing channel in this case is given by (19).

Consider now how to prove the implication " $\Leftarrow "$ in the third statement in (20). In this case one can construct Bosonic linear reversing channels for families of all states supported by particular subspaces of $\mathcal{H}_{A}$.

Indeed, if $Z_{B}^{0}$ is a nontrivial symplectic subspace of $Z_{f}$ then the second assertion of Lemma 2 below shows that the restriction $K^{0}$ of the operator $K$ to the subspace $Z_{B}^{0}$ is a symplectic embedding of this subspace into $Z_{A}$. Let $Z_{A}^{0}=K\left(Z_{B}^{0}\right)$. Then $Z_{X}=Z_{X}^{0} \oplus Z_{X}^{*}$, where $Z_{X}^{*}=\left[Z_{X}^{0}\right]^{\perp}$, and hence $\mathcal{H}_{X}=\mathcal{H}_{X}^{0} \otimes \mathcal{H}_{X}^{*}, X=A, B$. Let $\sigma$ be a given arbitrary state in $\mathfrak{S}\left(\mathcal{H}_{A}^{*}\right)$ and $\mathfrak{S}_{\sigma}=\left\{\rho \otimes \sigma \mid \rho \in \mathfrak{S}\left(\mathcal{H}_{A}^{0}\right)\right\} \subset \mathfrak{S}\left(\mathcal{H}_{A}\right)$. Let $\Psi^{0}(\cdot)=U_{K^{0}}(\cdot) U_{K^{0}}^{*}$ be a channel from $\mathfrak{T}\left(\mathcal{H}_{B}^{0}\right)$ to $\mathfrak{T}\left(\mathcal{H}_{A}^{0}\right)$, where $U_{K^{0}}$ is the unitary operator from $\mathcal{H}_{B}^{0}$ onto $\mathcal{H}_{A}^{0}$ implementing the 
symplectic transformation $K^{0}: Z_{B}^{0} \rightarrow Z_{A}^{0}$, and $\Psi^{*}$ be the completely depolarizing channel from $\mathfrak{T}\left(\mathcal{H}_{B}^{*}\right)$ to $\mathfrak{T}\left(\mathcal{H}_{A}^{*}\right)$ with the output state $\sigma$. Then $\Psi^{0} \otimes \Psi^{*}$ is a Bosonic linear channel from $\mathfrak{T}\left(\mathcal{H}_{B}\right)$ to $\mathfrak{T}\left(\mathcal{H}_{A}\right)$ and it is easy to see that $\Psi^{0} \otimes \Psi^{*}\left(\Phi_{K, f}(\omega)\right)=\omega$ for all $\omega \in \mathfrak{S}_{\sigma}$.

Lemma 2. Let $T: Z_{B} \oplus Z_{E} \rightarrow Z_{A} \oplus Z_{D}$ be a symplectic transformation defined by matrix (14). Then $[\operatorname{Ran} L]^{\perp}=K\left(\operatorname{ker} K_{D}\right)$ and $\operatorname{ker} K_{D}=\Delta_{B} K^{\top} \Delta_{A}\left([\operatorname{Ran} L]^{\perp}\right)$, where $[\operatorname{Ran} L]^{\perp}$ is the skew-orthogonal complement to the subspace $\operatorname{Ran} L=\{L z\}_{z \in Z_{E}} \subseteq Z_{A}$ (see Appendix B) $\mathrm{G}$

The restriction of the operator $K$ (correspondingly, $\Delta_{B} K^{\top} \Delta_{A}$ ) to the subspace ker $K_{D}$ (correspondingly, $[\operatorname{Ran} L]^{\perp}$ ) is nondegenerate and symplectic, i.e. it preserves the corresponding skew-symmetric forms $\Delta_{X}, X=A, B$.

This lemma shows that for a given Bosonic linear channel $\Phi_{K, f}$ the subspace $\operatorname{Ran} L$ is determined by the set $Z_{f}=\operatorname{ker} K_{D}$ and does not depend on a choice of its unitary dilation (13) -(14). It implies that the algebra generated by the Weyl operators $W_{A}(z), z \in K\left(Z_{f}\right)^{\perp}$, coincides with the noncommutative graph of the channel $\Phi_{K, f}$ (in terms of [8]).

Proof. Note first that $[\operatorname{Ran} L]^{\perp}=\operatorname{ker}\left[L^{\top} \Delta_{A}\right]$.

Since the matrix $T$ defined in (14) is symplectic, we have (cf. [13])

$$
\begin{aligned}
\Delta_{B} & =K^{\top} \Delta_{A} K+K_{D}^{\top} \Delta_{D} K_{D}, \\
0 & =L^{\top} \Delta_{A} K+L_{D}^{\top} \Delta_{D} K_{D}, \\
\Delta_{E} & =L^{\top} \Delta_{A} L+L_{D}^{\top} \Delta_{D} L_{D} .
\end{aligned}
$$

Since the group of symplectic matrices is closed under transposition, the matrix $T^{\top}$ is symplectic and hence we have the following equations (similar to (21))

$$
\begin{aligned}
\Delta_{A} & =K \Delta_{B} K^{\top}+L \Delta_{E} L^{\top}, \\
0 & =K_{D} \Delta_{B} K^{\top}+L_{D} \Delta_{E} L^{\top}, \\
\Delta_{D} & =K_{D} \Delta_{B} K_{D}^{\top}+L_{D} \Delta_{E} L_{D}^{\top} .
\end{aligned}
$$

The second equations in (21) and (22) imply respectively

$$
K\left(\operatorname{ker} K_{D}\right) \subseteq \operatorname{ker}\left[L^{\top} \Delta_{A}\right], \quad \Delta_{B} K^{\top} \Delta_{A}\left(\operatorname{ker}\left[L^{\top} \Delta_{A}\right]\right) \subseteq \operatorname{ker} K_{D}
$$

while the first equations in (21) and (22) show that

$$
\operatorname{ker} K \cap \operatorname{ker} K_{D}=\{0\}, \quad \operatorname{ker}\left[\Delta_{B} K^{\top} \Delta_{A}\right] \cap \operatorname{ker}\left[L^{\top} \Delta_{A}\right]=\{0\},
$$

since the matrices $\Delta_{A}$ and $\Delta_{B}$ are nondegenerate. It follows, by the dimension arguments, that " = " holds in the both inclusions in (23).

The last assertions of the lemma directly follow from the first equations in (21) and (22).

Corollary 3. If $\operatorname{det}\left[\Delta_{B}-K^{\top} \Delta_{A} K\right] \neq 0$ then $\operatorname{ri}\left(\Phi_{K, f}\right)=00$, i.e. the channel $\Phi_{K, f}$ is not reversible with respect to any families of pure states.

Proof. It is shown in [13] that the condition $\operatorname{det}\left[\Delta_{B}-K^{\top} \Delta_{A} K\right] \neq 0$ implies existence of unitary dilation (13)-(14) for the channel $\Phi_{K, f}$ in which $D=B$ and $K_{D}$ is a nondegenerate quadratic matrix.

\footnotetext{
${ }^{7}$ We will always use this sense of the symbol " $\perp$ " dealing with a subspace of a symplectic space.
} 


\section{IV.2 The case of Gaussian channels}

Bosonic Gaussian channels are Bosonic linear channels defined by (12) with the function

$$
f(z)=\exp \left(\mathrm{i} l z-\frac{1}{2} z^{\top} \alpha z\right)
$$

where $l$ is a $2 s_{B}$-dimensional real row and $\alpha$ is a real symmetric $\left(2 s_{B}\right) \times\left(2 s_{B}\right)$ matrix satisfying the inequality $\alpha \geq \pm \frac{i}{2}\left[\Delta_{B}-K^{\top} \Delta_{A} K\right]$ [3, 9, 12].

Any such channel can be transformed by appropriate displacement unitaries to the Bosonic Gaussian channel with $l=0$ and the same matrix $\alpha$ for which unitary dilation (13)-(14) always exists with Gaussian state $\rho_{D}$ [3, 12]. In this case $\alpha=K_{D}^{\top} \alpha_{D} K_{D}$, where $\alpha_{D}$ is the covariance matrix of $\rho_{D}$. Thus all the above results can be reformulated for Bosonic Gaussian channels by noting that $Z_{f}=\operatorname{ker} K_{D}=\operatorname{ker} \alpha$ (since the matrix $\alpha_{D}$ is nondegenerate). In particular, Theorem 3 is reformulated as follows.

Corollary 4. Let $\Phi$ be a noisy (not noiseless [27]) Bosonic Gaussian channel with the parameters $K, l, \alpha$. Then

$$
\begin{aligned}
& \{\operatorname{ri}(\Phi)=00\} \Leftrightarrow\{\operatorname{det} \alpha \neq 0\}, \\
& \{\operatorname{ri}(\Phi)=01\} \Leftrightarrow\left\{\operatorname{ker} \alpha \text { is a nontrivial isotropic subspace of } Z_{B}\right\}, \\
& \{\operatorname{ri}(\Phi)=02\} \Leftrightarrow\left\{\exists z_{1}, z_{2} \in \operatorname{ker} \alpha \text { such that } \Delta_{B}\left(z_{1}, z_{2}\right) \neq 0\right\} .
\end{aligned}
$$

Physically, this characterization of reversibility of a Gaussian channel $\Phi$ is intuitively clear, since in the Heisenberg picture the condition $\operatorname{det} \alpha \neq 0$ means that the channel $\Phi^{*}$ injects quantum noise in all canonical variables of the system $B$, while degeneracy of the matrix $\alpha$ is equivalent to existence of noise-free canonical variables. Corollary 4 shows that

- the channel $\Phi$ is reversible with respect to some families of pure states if and only if the set of noise-free canonical variables is nonempty;

- the channel $\Phi$ is reversible only with respect to some orthogonal families of pure states if and only if all noise-free canonical variables commute;

- the channel $\Phi$ is reversible with respect to some nonorthogonal families of pure states (and hence it is perfectly reversible on a particular subspace) if and only if there are noncommuting noise-free canonical variables.

Example: one-mode Gaussian channels. The simplest Bosonic Gaussian channels are one-mode channels for which $\operatorname{dim} Z_{A}=\operatorname{dim} Z_{B}=2$.

A classification of all one-mode Gaussian channels is obtained in [14, where it is shown that there exist the following canonical types

$$
A_{1}[N], \quad A_{2}[N], \quad B_{1}, \quad B_{2}[N], \quad C[k, N](k>0, k \neq 1), \quad D[k, N](k>0)
$$

of such channels (the parameter $N \geq 0$ denotes the level of noise, see details in [12, 14]). 
By Corollary 4 all one-mode Gaussian channels are not reversible with respect to any families of pure states excepting the noiseless channel $B_{2}[0]$ and the channel $B_{1}$ which has reversibility index 01 . All reversed families of pure states for the channel $B_{1}$ are described in Section IV.3.

By Proposition 2 the channel $B_{1}$ is the only noisy one-mode Gaussian channel reversible with respect to some complete orthogonal families of (infinite rank) states. Applying the proof of Proposition 2 to the channel $B_{1}$ we have $\mathcal{A}=\mathcal{B}=L_{\infty}(\mathbb{R})$ and hence any orthogonal resolution of the identity $\left\{P_{i}\right\}$ in $\mathcal{A}$ corresponds to a decomposition $\left\{D_{i}\right\}$ of $\mathbb{R}$ into disjoint measurable subsets. In this case $\left\{Q_{i}\right\}=\left\{P_{i}\right\}$ and $\mathcal{H}_{A}^{i}=\mathcal{H}_{B}^{i}=L_{2}\left(D_{i}\right)$ is the subspace of $\mathcal{H}_{A}=\mathcal{H}_{B}=L_{2}(\mathbb{R})$ consisting of functions supported by $D_{i}$.

Thus, the subset $\mathfrak{S}\left(L_{2}\left(D_{i}\right)\right) \subset \mathfrak{S}\left(L_{2}(\mathbb{R})\right)$ is mapped by the channel $B_{1}$ into itself for each $i$. This conclusion agrees with the explicit formula for the channel $B_{1}$ (formula (7.1) in [16] with $q$ replaced by $p$ ).

It follows that the channel $B_{1}$ is reversible with respect to any family of states $\left\{\rho_{i}\right\}$ such that $\rho_{i} \in \mathfrak{S}\left(L_{2}\left(D_{i}\right)\right)$ for each $i$. One can expect that all reversed families for the channel $B_{1}$ have such form. For families of pure states this is proved in Section IV.3 (see the example).

In regard to reversibility of one-mode Gaussian channels with respect to nonorthogonal families of mixed states we have the following partial result.

Corollary 5. Let $\Phi$ be a one-mode Gaussian channels of any type excepting $B_{1}, B_{2}[0]$ and $C[k, 0]$ with $k>1$. Then the channel $\Phi$ is not reversible with respect to any complete family of states containing at least one finite rank state.

Proof. As shown in [16] all operators of any Kraus representation of the channel $C[k, 0]$ with $k \neq 1$ have infinite rank. By Theorem 1 (see Remark 2) this implies nonreversibility of the complementary channel to the channel $C[k, 0]$ with $k \neq 1$ with respect to any complete family of states containing at least one finite rank state. This implies nonreversibility of the channels $C[k, 0]$ with $k<1$ and $D[k, 0]$ (complementary channels to one-mode Gaussian channels are described in [12, 14]). Nonreversibility of all the others channels excepting the channels $B_{1}, B_{2}[0]$ and $C[k, 0]$ with $k>1$ can be shown by using their representation in the form $\Psi \circ \Phi$, where $\Phi$ is either the channel $C[k, 0]$ with $k<1$ or the channel $D[k, 0]$ (see Table I in [16]).

\section{IV.3 Explicit forms of reversed families}

Now we will give an explicit description of reversed families of pure states for the channel $\Phi_{K, f}$. We will show that these families are completely determined by the subspace $K\left(Z_{f}\right)$ of $Z_{A}$. By Theorem 3 it suffices to consider the cases $\operatorname{ri}\left(\Phi_{K, f}\right)=01,02$.

$\operatorname{ri}\left(\Phi_{\mathbf{K}, \mathbf{f}}\right)=\mathbf{0 1}$. By Theorem 3 and Lemma 2 in this case $K\left(Z_{f}\right)$ is a nontrivial isotropic subspace of $Z_{A}$ and hence the subspace $\operatorname{Ran} L=\left[K\left(Z_{f}\right)\right]^{\perp}$ contains a maximal isotropic 
subspace of $Z_{A}$. By Lemma [6 in Appendix B there exists a symplectic basis $\left\{\tilde{e}_{k}, \tilde{h}_{k}\right\}$ in $Z_{A}$ such that $\left\{\tilde{e}_{1}, \ldots, \tilde{e}_{s_{A}}, \tilde{h}_{d+1}, \ldots, \tilde{h}_{s_{A}}\right\}$ is a basis in $\operatorname{Ran} L, 0<d \leq s_{A}$. If we identify the space $\mathcal{H}_{A}$ with the space $L_{2}\left(\mathbb{R}^{s_{A}}\right)$ of complex-valued functions of $s_{A}$ variables (which will be denoted $\left.\xi_{1}, \ldots, \xi_{s_{A}}\right)$ and the Weyl operators $W_{A}\left(\tilde{e}_{k}\right)$ and $W_{A}\left(\tilde{h}_{k}\right)$ with the operators

$$
\psi\left(\xi_{1}, \ldots, \xi_{s_{A}}\right) \mapsto e^{\mathrm{i} \xi_{k}} \psi\left(\xi_{1}, \ldots, \xi_{s_{A}}\right) \text { and } \psi\left(\xi_{1}, \ldots, \xi_{s_{A}}\right) \mapsto \psi\left(\xi_{1}, \ldots, \xi_{k}+1, \ldots, \xi_{s_{A}}\right)
$$

then Theorem 2, Lemma 3 and the proof of Proposition 3 in Appendix A show that all reversed families of pure states for the channel $\Phi_{K, f}$ correspond to families $\left\{\psi_{i}\right\}$ of functions in $L_{2}\left(\mathbb{R}^{s_{A}}\right)$ with unit norm satisfying the following condition

$$
\psi_{i} \cdot S_{y_{d+1}, \ldots, y_{s}} \psi_{j}=0\left(\text { in } L_{2}\left(\mathbb{R}^{s_{A}}\right)\right) \quad \forall\left(y_{d+1}, \ldots, y_{s_{A}}\right) \in \mathbb{R}^{s_{A}-d}, \forall i \neq j,
$$

where $S_{y_{d+1}, \ldots, y_{s_{A}}}$ is a shift operator by the vector $\left(0, \ldots, 0, y_{d+1}, \ldots, y_{s_{A}}\right)$ :

$$
\left(S_{y_{d+1}, \ldots, y_{s_{A}}} \psi\right)\left(\xi_{1}, \ldots, \xi_{s_{A}}\right)=\psi\left(\xi_{1}, \ldots, \xi_{d}, \xi_{d+1}+y_{d+1}, \ldots, \xi_{s_{A}}+y_{s_{A}}\right) .
$$

This condition means, roughly speaking, that all shifts in $\mathbb{R}^{s_{A}}$ of the supports of the functions of the family $\left\{\psi_{i}\right\}$ along the last $s_{A}-d$ coordinates do not intersect each other.

As an example of a reversed family one can take the family of product pure states $\left|\phi_{i} \otimes \varphi\right\rangle\left\langle\phi_{i} \otimes \varphi\right|$ corresponding to the family of functions

$$
\psi_{i}\left(\xi_{1}, \ldots, \xi_{s_{A}}\right)=\phi_{i}\left(\xi_{1}, \ldots, \xi_{d}\right) \varphi\left(\xi_{d+1}, \ldots, \xi_{s_{A}}\right)
$$

where $\left\{\phi_{i}\right\}$ is a family of functions in $L_{2}\left(\mathbb{R}^{d}\right)$ with mutually disjoint supports and $\varphi$ is a given function in $L_{2}\left(\mathbb{R}^{s_{A}-d}\right)$.

Example: one-mode Gaussian channel $B_{1}$. In this case $s_{A}=s_{B}=1$,

$$
K=\left[\begin{array}{ll}
1 & 0 \\
0 & 1
\end{array}\right], \quad \alpha=\left[\begin{array}{cc}
0 & 0 \\
0 & 1 / 4
\end{array}\right], \quad Z_{f}=\operatorname{ker} \alpha=\left[\begin{array}{l}
1 \\
0
\end{array}\right] \text {. }
$$

Hence $\tilde{e}_{1}=[1,0]^{\top}, \tilde{h}_{1}=[0,1]^{\top}\left(\right.$ since $\left.K\left(Z_{f}\right)^{\perp}=K\left(Z_{f}\right)=\left\{\lambda \tilde{e}_{1}\right\}\right)$ and condition (24) shows that all reversed families of pure states for this channel have the form

$$
\left\{\left|\psi_{i}\right\rangle\left\langle\psi_{i}\right|\right\} \text {, where }\left\{\psi_{i}\right\} \subset L_{2}(\mathbb{R}) \text { such that } \psi_{i} \cdot \psi_{j}=0\left(\text { in } L_{2}(\mathbb{R})\right) \forall i \neq j \text {. }
$$

i.e., roughly speaking, all reversed families of pure states correspond to families of functions with mutually disjoint supports (in agreement with the observations in Section IV.2 which show sufficiency of this condition).

$\operatorname{ri}\left(\Phi_{\mathbf{K}, \mathbf{f}}\right)=02$. By Theorem 3 in this case there exists a symplectic subspace $Z_{B}^{0}$ of $Z_{f}$. By Lemma $2 Z_{A}^{0}=K\left(Z_{B}^{0}\right)$ is a symplectic subspace of $[\operatorname{Ran} L]^{\perp}=K\left(Z_{f}\right)$. Let $\left\{\tilde{e}_{k}, \tilde{h}_{k}\right\}_{k=1}^{s_{A}}$ be a symplectic basis in $Z_{A}$ such that $\left\{\tilde{e}_{k}, \tilde{h}_{k}\right\}_{k=1}^{d}$ is a symplectic basis in $Z_{A}^{0}$. If we identify the space $\mathcal{H}_{A}$ with the space $L_{2}\left(\mathbb{R}^{s_{A}}\right)$ as before then Theorem 2 , Lemma 3 and the proof of Proposition 3 in Appendix A show that the channel $\Phi_{K, f}$ is perfectly reversible on the subspaces $L_{2}\left(\mathbb{R}^{d}\right) \otimes\{c|\varphi\rangle\}, \varphi \in L_{2}\left(\mathbb{R}^{s_{A}-d}\right.$ ) (in agreement with the second part of Remark 6). 


\section{Applications}

By Petz's theorem reversibility properties of a quantum channel are closely related to the question of (non)-preserving the Holevo quantity of arbitrary (discrete or continuous) ensembles of states under action of this channel, i.e. to the question of validity of an equality in the general inequality

$$
\chi(\Phi(\mu)) \leq \chi(\mu)
$$

which holds, by monotonicity of the relative entropy, for any channel $\Phi: \mathfrak{T}\left(\mathcal{H}_{A}\right) \rightarrow \mathfrak{T}\left(\mathcal{H}_{B}\right)$ and any generalized ensemble $\mu$ of states in $\mathfrak{S}\left(\mathcal{H}_{A}\right)$ (defined as a Borel probability measure on $\mathfrak{S}\left(\mathcal{H}_{A}\right)$, see [25, Section 5]).

Denote by $\mathcal{P}\left(\mathfrak{S}\left(\mathcal{H}_{A}\right)\right)$ the set of all generalized ensembles of pure states (probability measures on $\mathfrak{S}\left(\mathcal{H}_{A}\right)$ supported by pure states $)$. Denote by $\mathcal{P}_{\mathrm{c}}\left(\mathfrak{S}\left(\mathcal{H}_{A}\right)\right)$ and $\mathcal{P}_{\mathrm{o}}\left(\mathfrak{S}\left(\mathcal{H}_{A}\right)\right)$ the subsets of $\mathcal{P}\left(\mathfrak{S}\left(\mathcal{H}_{A}\right)\right)$ consisting respectively of all ensembles with nondegenerate average state (barycenter) and of all ensembles of mutually orthogonal pure states.

For a given channel $\Phi: \mathfrak{T}\left(\mathcal{H}_{A}\right) \rightarrow \mathfrak{T}\left(\mathcal{H}_{B}\right)$ let $\mathcal{P}(\Phi)$ be the subset of $\mathcal{P}\left(\mathfrak{S}\left(\mathcal{H}_{A}\right)\right)$ consisting of all ensembles $\mu$ for which an equality holds in (25). The version of Petz's theorem for continuous ensembles (Proposition 3 in [25]) shows that:

$$
\begin{aligned}
& \{\operatorname{ri}(\Phi)=00\} \Rightarrow\{\mathcal{P}(\Phi)=\emptyset\}, \\
& \{\operatorname{ri}(\Phi)=01\} \Rightarrow\left\{\mathcal{P}(\Phi) \subset \mathcal{P}_{\mathrm{o}} \backslash \mathcal{P}_{\mathrm{c}}\right\} \\
& \{\operatorname{ri}(\Phi)=02\} \Rightarrow\left\{\mathcal{P}(\Phi) \subset \mathcal{P} \backslash \mathcal{P}_{\mathrm{c}}\right\} \\
& \{\operatorname{ri}(\Phi)=11\} \Rightarrow\left\{\mathcal{P}(\Phi) \subset \mathcal{P}_{\mathrm{o}}\right\} \\
& \{\operatorname{ri}(\Phi)=12\} \Rightarrow\left\{\mathcal{P}(\Phi) \subset \mathcal{P}_{\mathrm{o}} \cup\left[\mathcal{P} \backslash \mathcal{P}_{\mathrm{c}}\right]\right\},
\end{aligned}
$$

where we write $\mathcal{P}_{*}$ instead of $\mathcal{P}_{*}\left(\mathfrak{S}\left(\mathcal{H}_{A}\right)\right)$ for brevity.

Thus, Corollary 4 implies the following assertions.

Corollary 6. Let $\Phi$ be a Gaussian channel with the parameters $K, l, \alpha$.

A) If $\Phi$ is not a noiseless channel then

$$
\chi(\Phi(\mu))<\chi(\mu)
$$

for any ensemble $\mu$ of pure states with nondegenerate average state;

B) If $\operatorname{ker} \alpha$ is an isotropic subspace of $Z_{B}$ then (26) holds for any nonorthogonal (in particular, continuous) ensemble $\mu$ of pure states;

C) If $\operatorname{det} \alpha \neq 0$ then (26) holds for any ensemble $\mu$ of pure states.

By using the observations in Section IV.3 one can describe all ensembles $\mu$ of pure states for which $\chi(\Phi(\mu))=\chi(\mu)$. All such ensembles are completely determined by the subspace $K(\operatorname{ker} \alpha)$.

Some applications of conditions for an equality (strict inequality) in (25) to study of capacities of quantum channels are considered in [25, Section 5]. 


\section{Acknowledgments}

I am grateful to A.S.Holevo and to the participants of his seminar "Quantum probability, statistic, information" (the Steklov Mathematical Institute) for useful discussion. I am also grateful to the referee for suggestions improving the paper. The work is partially supported by the RAS research program and by RFBR grants 12-01-00319a and 13-01-00295a.

\section{Appendix A: On discrete c-q subchannels and completely depolarizing subchannels of Bosonic linear channels}

Note first that any nontrivial Bosonic linear channel $\Phi_{K, f}$ is not a discrete c-q channel (it is a discrete c-q channel if and only if it is completely depolarizing). This immediately follows from Definition 2 and (12), since the Weyl operator $W_{A}(K z)$ has purely point spectrum for any $z \in Z_{B}$ if and only if $K=0$.

In this section we explore necessary and sufficient conditions for existence of discrete c-q subchannels and of completely depolarizing subchannels of a Bosonic linear channel $\Phi_{K, f}$.

The following lemma shows that all discrete c-q subchannels and all completely depolarizing subchannels of a quantum channel are determined by its kernel (null set).

Lemma 3. Let $\Psi: \mathfrak{T}\left(\mathcal{H}_{A}\right) \rightarrow \mathfrak{T}\left(\mathcal{H}_{B}\right)$ be a quantum channel and $\Pi(\Psi)$ the set of all families $\left\{\left|\psi_{i}\right\rangle\right\}$ of unit vectors in $\mathcal{H}_{A}$ such that $\Psi\left(\left|\psi_{i}\right\rangle\left\langle\psi_{j}\right|\right)=0$ for all $i \neq j$. The channel $\Psi$ has a discrete $c-q$ subchannel corresponding to a subspace $\mathcal{H}_{0}$, i.e.

$$
\Psi(\rho)=\sum_{i}\left\langle\psi_{i}|\rho| \psi_{i}\right\rangle \sigma_{i} \quad \forall \rho \in \mathfrak{T}\left(\mathcal{H}_{0}\right)
$$

where $\left\{\left|\psi_{i}\right\rangle\right\}$ is an orthonormal basis in $\mathcal{H}_{0}$, if and only if $\left\{\left|\psi_{i}\right\rangle\right\} \in \Pi(\Psi)$. Under this condition

$$
\sigma_{i}=\sigma_{j} \quad \Leftrightarrow \quad \Psi\left(\left|\psi_{i}\right\rangle\left\langle\psi_{i}|-| \psi_{j}\right\rangle\left\langle\psi_{j}\right|\right)=0
$$

Proof. It suffices to note that $\rho=\sum_{i, j}\left\langle\psi_{i}|\rho| \psi_{j}\right\rangle\left|\psi_{i}\right\rangle\left\langle\psi_{j}\right|$ for any $\rho \in \mathfrak{T}\left(\mathcal{H}_{0}\right)$.

Remark 7. The conditions $\Psi\left(\left|\psi_{i}\right\rangle\left\langle\psi_{j}\right|\right)=0$ and $\Psi\left(\left|\psi_{i}\right\rangle\left\langle\psi_{i}\right|\right)=\Psi\left(\left|\psi_{j}\right\rangle\left\langle\psi_{j}\right|\right)$ can be expressed respectively as follows

$$
\left\langle\psi_{i}\left|\Psi^{*}(B)\right| \psi_{j}\right\rangle=0 \quad \forall B \in \mathfrak{B}\left(\mathcal{H}_{B}\right), \quad\left\langle\psi_{i}\left|\Psi^{*}(B)\right| \psi_{i}\right\rangle=\left\langle\psi_{j}\left|\Psi^{*}(B)\right| \psi_{j}\right\rangle \quad \forall B \in \mathfrak{B}\left(\mathcal{H}_{B}\right),
$$

where $\Psi^{*}: \mathfrak{B}\left(\mathcal{H}_{B}\right) \rightarrow \mathfrak{B}\left(\mathcal{H}_{A}\right)$ is a dual map to the channel $\Psi$.

If $\Psi=\Phi_{K, f}$ then, since the family $\left\{W_{B}(z)\right\}_{z \in Z_{B}}$ generates $\mathfrak{B}\left(\mathcal{H}_{B}\right)$ and $f$ is a continuous function such that $f(0)=1$, the above conditions can be rewritten as

$$
\begin{gathered}
\left\langle\psi_{i}\left|W_{A}(K z)\right| \psi_{j}\right\rangle=0 \quad \forall z \in Z_{B} \\
\left\langle\psi_{i}\left|W_{A}(K z)\right| \psi_{i}\right\rangle=\left\langle\psi_{j}\left|W_{A}(K z)\right| \psi_{j}\right\rangle \quad \forall z \in Z_{B} .
\end{gathered}
$$


By using Remark 7 it is easy to show that the set $\Pi\left(\Phi_{K, f}\right)$ introduced in Lemma 3 is empty if and only if $\operatorname{rank} K=\operatorname{dim} Z_{A}$ and to describe all families belonging to this set in the case $\operatorname{rank} K<\operatorname{dim} Z_{A}$. This will be done in the proof of the following proposition.

Proposition 3. The channel $\Phi_{K, f}$ has discrete $c-q$ subchannels if and only if $\operatorname{rank} K<$ $\operatorname{dim} Z_{A}$. Under this condition all these subchannels are not completely depolarizing if and only if

$$
\operatorname{Ran} K \doteq\{K z\}_{z \in Z_{B}} \text { contains a maximal isotropic subspace of } Z_{A} \text {, }
$$

which means that the subspace $[\operatorname{Ran} K]^{\perp}$ is isotropic, i.e. there exist no $z_{1}, z_{2} \in \operatorname{ker}\left[K^{\top} \Delta_{A}\right]$ such that $\Delta_{A}\left(z_{1}, z_{2}\right) \neq 0$.

Proof. If $\operatorname{rank} K=\operatorname{dim} Z_{A}$ then the family $\left\{W_{A}(K z)\right\}_{z \in Z_{B}}$ of Weyl operators acts irreducibly on $\mathcal{H}_{A}$. Hence condition (A-1) can not be valid.

If $\operatorname{rank} K<\operatorname{dim} Z_{A}$ and condition (A-3) holds then Lemma 6 in Appendix B implies existence of a symplectic basis $\left\{\tilde{e}_{k}, \tilde{h}_{k}\right\}$ in $Z_{A}$ such that $\left\{\tilde{e}_{1}, \ldots, \tilde{e}_{s_{A}}, \tilde{h}_{d+1}, \ldots, \tilde{h}_{s_{A}}\right\}$ is a basis in $\operatorname{Ran} K, d \leq s_{A}$. Let $Z_{B}^{0}$ be a subspace of $Z_{B}$ with the basis $\left\{z_{1}^{e}, \ldots, z_{s_{A}}^{e}, z_{d+1}^{h}, \ldots, z_{s_{A}}^{h}\right\}$ such that $\tilde{e}_{k}=K z_{k}^{e}$ for all $k=\overline{1, s_{A}}$ and $\tilde{h}_{k}=K z_{k}^{h}$ for all $k=\overline{d+1, s_{A}}$. Thus for any vector $z \in Z_{B}^{0}$ represented as $z=\sum_{k=1}^{s_{A}} x_{k} z_{k}^{e}+\sum_{k=d+1}^{s_{A}} y_{k} z_{k}^{h},\left(x_{1}, \ldots, x_{s_{A}}\right) \in \mathbb{R}^{s_{A}},\left(y_{d+1}, \ldots, y_{s_{A}}\right) \in \mathbb{R}^{s_{A}-d}$ we have

$$
\begin{gathered}
W_{A}(K z)=W_{A}\left(\sum_{k=1}^{s_{A}} x_{k} K z_{k}^{e}+\sum_{k=d+1}^{s_{A}} y_{k} K z_{k}^{h}\right)=W_{A}\left(\sum_{k=1}^{s_{A}} x_{k} \tilde{e}_{k}+\sum_{k=d+1}^{s_{A}} y_{k} \tilde{h}_{k}\right) \\
=\lambda W_{A}\left(x_{1} \tilde{e}_{1}\right) \cdot \ldots \cdot W_{A}\left(x_{s_{A}} \tilde{e}_{s_{A}}\right) \cdot W_{A}\left(y_{d+1} \tilde{h}_{d+1}\right) \cdot \ldots \cdot W_{A}\left(y_{s_{A}} \tilde{h}_{s_{A}}\right),
\end{gathered}
$$

where $\lambda=e^{\mathrm{i}\left[x_{d+1} y_{d+1}+\ldots+x_{s_{A}} y_{s_{A}}\right]} \neq 0$.

By identifying the space $\mathcal{H}_{A}$ with the space $L_{2}\left(\mathbb{R}^{s_{A}}\right)$ of complex-valued functions of $s_{A}$ variables (which will be denoted $\left.\xi_{1}, \ldots, \xi_{s_{A}}\right)$ and the Weyl operators $W_{A}\left(\tilde{e}_{k}\right)$ and $W_{A}\left(\tilde{h}_{k}\right)$ with the operators

$$
\psi\left(\xi_{1}, \ldots, \xi_{s_{A}}\right) \mapsto e^{\mathrm{i} \xi_{k}} \psi\left(\xi_{1}, \ldots, \xi_{s_{A}}\right) \text { and } \psi\left(\xi_{1}, \ldots, \xi_{s_{A}}\right) \mapsto \psi\left(\xi_{1}, \ldots, \xi_{k}+1, \ldots, \xi_{s_{A}}\right)
$$

the equality in (A-1) for the vector $z$ can be rewritten as follows

$$
\int \overline{\psi_{i}\left(\xi_{1}, \ldots, \xi_{s_{A}}\right)}\left(S_{y_{d+1}, \ldots, y_{s_{A}}} \psi_{j}\right)\left(\xi_{1}, \ldots, \xi_{s_{A}}\right) e^{\mathrm{i}\left(x_{1} \xi_{1}+\ldots+x_{s_{A}} \xi_{s_{A}}\right)} d \xi_{1}, \ldots, d \xi_{s_{A}}=0
$$

where $\left(S_{y_{d+1}, \ldots, y_{s_{A}}} \psi_{j}\right)\left(\xi_{1}, \ldots, \xi_{s_{A}}\right)=\psi_{j}\left(\xi_{1}, \ldots, \xi_{d}, \xi_{d+1}+y_{d+1}, \ldots, \xi_{s_{A}}+y_{s_{A}}\right)$.

This equality is valid for all $\left(x_{1}, \ldots, x_{s_{A}}\right) \in \mathbb{R}^{s_{A}}$ and $\left(y_{d+1}, \ldots, y_{s_{A}}\right) \in \mathbb{R}^{s_{A}-d}$ (that is for all $z \in Z_{B}^{0}$ ) if and only if

$$
\overline{\psi_{i}\left(\xi_{1}, \ldots, \xi_{s_{A}}\right)}\left(S_{y_{d+1}, \ldots, y_{s_{A}}} \psi_{j}\right)\left(\xi_{1}, \ldots, \xi_{s_{A}}\right)=0
$$

for almost all $\left(\xi_{1}, \ldots, \xi_{s_{A}}\right) \in \mathbb{R}^{s_{A}}$ and all $\left(y_{d+1}, \ldots, y_{s_{A}}\right) \in \mathbb{R}^{s_{A}-d}$. Since $\operatorname{Ran} K=K\left(Z_{B}^{0}\right)$, it implies that the set $\Pi\left(\Phi_{K, f}\right)$ introduced in Lemma 3 consists of families $\left\{\psi_{i}\right\}$ satisfying the condition

$$
\psi_{i} \cdot S_{y_{d+1}, \ldots, y_{s_{A}}} \psi_{j}=0\left(\text { in } L_{2}\left(\mathbb{R}^{s_{A}}\right)\right) \quad \forall\left(y_{d+1}, \ldots, y_{s_{A}}\right) \in \mathbb{R}^{s_{A}-d}, \forall i \neq j .
$$


This condition means, roughly speaking, that all shifts in $\mathbb{R}^{s_{A}}$ of the supports of the functions of the family $\left\{\psi_{i}\right\}$ along the last $s_{A}-d$ coordinates do not intersect each other.

As an example of a family satisfying condition (A-4) one can take the family of functions

$$
\psi_{i}\left(\xi_{1}, \ldots, \xi_{s_{A}}\right)=\phi_{i}\left(\xi_{1}, \ldots, \xi_{d}\right) \varphi\left(\xi_{d+1}, \ldots, \xi_{s_{A}}\right)
$$

where $\left\{\phi_{i}\right\}$ is a family of functions in $L_{2}\left(\mathbb{R}^{d}\right)$ with mutually disjoint supports and $\varphi$ is a given function in $L_{2}\left(\mathbb{R}^{s_{A}-d}\right)$. It is clear that this family (consisting of tensor product vectors $\left.\left|\phi_{i} \otimes \varphi\right\rangle\right)$ is not a "general solution" of (A-4).

To show that for any $i \neq j$ the equality in (A-2) can not be valid for all $z \in Z_{B}$ note that this equality for the vector $z=\sum_{k=1}^{s_{A}} x_{k} z_{k}^{e} \in Z_{B}^{0}$ can be rewritten as follows

$$
\begin{aligned}
& \int\left|\psi_{i}\left(\xi_{1}, \ldots, \xi_{s_{A}}\right)\right|^{2} e^{\mathrm{i}\left(x_{1} \xi_{1}+\ldots+x_{s_{A}} \xi_{s_{A}}\right)} d \xi_{1}, \ldots, d \xi_{s_{A}} \\
= & \int\left|\psi_{j}\left(\xi_{1}, \ldots, \xi_{s_{A}}\right)\right|^{2} e^{\mathrm{i}\left(x_{1} \xi_{1}+\ldots+x_{s_{A}} \xi_{s_{A}}\right)} d \xi_{1}, \ldots, d \xi_{s_{A}} .
\end{aligned}
$$

Validity of this equality for all $\left(x_{1}, \ldots, x_{s_{A}}\right) \in \mathbb{R}^{s_{A}}$ means that the classical characteristic functions of the probability densities $\left|\psi_{i}\right|^{2}$ and $\left|\psi_{j}\right|^{2}$ coincide. But this obviously contradicts to condition (A-4).

If condition (A-3) is not valid then the subspace $[\operatorname{Ran} K]^{\perp}$ contains a symplectic subspace $Z_{A}^{0}$. Let $\left\{\tilde{e}_{k}, \tilde{h}_{k}\right\}_{k=1}^{s_{A}}$ be a symplectic basis in $Z_{A}$ such that $\left\{\tilde{e}_{k}, \tilde{h}_{k}\right\}_{k=1}^{d}$ is a symplectic basis in $Z_{A}^{0}$.

By identifying the space $\mathcal{H}_{A}$ with the space $L_{2}\left(\mathbb{R}^{s_{A}}\right)$ as before we see that the equalities in (A-1) and in (A-2) can be rewritten respectively as

$$
\int \overline{\psi_{i}\left(\xi_{1}, \ldots, \xi_{s_{A}}\right)}\left(W_{A}(K z) \psi_{j}\right)\left(\xi_{1}, \ldots, \xi_{s_{A}}\right) d \xi_{1}, \ldots, d \xi_{s_{A}}=0
$$

and

$$
\begin{aligned}
& \int \overline{\psi_{i}\left(\xi_{1}, \ldots, \xi_{s_{A}}\right)}\left(W_{A}(K z) \psi_{i}\right)\left(\xi_{1}, \ldots, \xi_{s_{A}}\right) d \xi_{1}, \ldots, d \xi_{s_{A}} \\
= & \int \overline{\psi_{j}\left(\xi_{1}, \ldots, \xi_{s_{A}}\right)}\left(W_{A}(K z) \psi_{j}\right)\left(\xi_{1}, \ldots, \xi_{s_{A}}\right) d \xi_{1}, \ldots, d \xi_{s_{A}},
\end{aligned}
$$

where $\left\{\psi_{i}\right\}$ is an orthonormal family of functions in $L_{2}\left(\mathbb{R}^{s_{A}}\right)$.

Since for any $z \in Z_{B}$ the vector $K z$ has no components corresponding to the vectors $\tilde{e}_{k}, \tilde{h}_{k}, k=\overline{1, d}$, one can satisfy equalities (A-6) and (A-7) for all $i \neq j$ and all $z \in Z_{B}$ by taking the family of functions (A-5), in which $\left\{\phi_{i}\right\}$ is an arbitrary orthonormal basis in $L_{2}\left(\mathbb{R}^{d}\right)$ and $\varphi$ is a given function in $L_{2}\left(\mathbb{R}^{s_{A}-d}\right)$. Thus this family belongs to the set $\Pi\left(\Phi_{K, f}\right)$ introduced in Lemma 3 and this lemma shows that the restriction of the channel $\Phi_{K, f}$ to any subspace of the form $L_{2}\left(\mathbb{R}^{d}\right) \otimes\{c|\varphi\rangle\}, \varphi \in L_{2}\left(\mathbb{R}^{s_{A}-d}\right)$, is completely depolarizing. 


\section{Appendix B: Some facts about symplectic spaces}

In what follows $Z$ is a $2 s$-dimensional symplectic space with the nondegenerate skew-symmetric form $\Delta[1,12,19]$. The set of vectors $\left\{e_{1}, \ldots, e_{s}, h_{1}, \ldots, h_{s}\right\}$ is called symplectic basis in $Z$ if $\Delta\left(e_{k}, e_{l}\right)=\Delta\left(h_{k}, h_{l}\right)=0$ for all $k, l$, but $\Delta\left(e_{k}, h_{l}\right)=\delta_{k l}$. For an arbitrary subspace $L \subset Z$ one can define its skew-orthogonal complement $L^{\perp}=\left\{z \in Z \mid \Delta\left(z, z^{\prime}\right)=0 \forall z^{\prime} \in L\right\}$. Despite the fact that $L \cap L^{\perp} \neq\{0\}$ in general, we always have the familiar relations

$$
\left[L^{\perp}\right]^{\perp}=L \quad \text { and } \quad \operatorname{dim} L+\operatorname{dim} L^{\perp}=\operatorname{dim} Z .
$$

A linear transformation $T: Z \rightarrow Z$ is called symplectic if $\Delta\left(T z_{1}, T z_{2}\right)=\Delta\left(z_{1}, z_{2}\right)$ for all $z_{1}, z_{2} \in Z$. A symplectic transformation maps any symplectic basis to symplectic basis and vice versa: any two symplectic base are related by the particular symplectic transformation.

A subspace $L$ of $Z$ is called symplectic if the form $\Delta$ is nondegenerate on $L$, in this case $L$ has even dimension and can be considered as a symplectic space of itself. We will use the following simple observation [1, 19].

Lemma 4. If $L$ is a symplectic subspace of $Z$ then $L^{\perp}$ is a symplectic subspace of $Z$ and $Z=L+L^{\perp}$ (i.e. $Z=L \vee L^{\perp}$ and $L \cap L^{\perp}=\{0\}$ ).

By joining the symplectic base in $L$ and in $L^{\perp}$ we obtain a symplectic basis in $Z$.

A subspace $L$ of $Z$ is called isotropic if the form $\Delta$ equals to zero on $L$. In this case $L$ has dimension $\leq s$. We will use the following observation.

Lemma 5. If $L$ is an isotropic subspace of $Z, \operatorname{dim} L=d$, then there exists a symplectic basis $\left\{\tilde{e}_{k}, \tilde{h}_{k}\right\}$ in $Z$ such that $\left\{\tilde{e}_{1}, \ldots, \tilde{e}_{d}\right\}$ is a basis in $L$.

Proof. Let $\left\{e_{k}, h_{k}\right\}$ be an arbitrary symplectic basis in $Z$ and $L^{\prime}$ the isotropic subspace of $Z$ generated by the vectors $e_{1}, \ldots, e_{d}$. Since the isotropic subspaces $L$ and $L^{\prime}$ have the same dimension, there is a symplectic transformation $T$ such that $L=T\left(L^{\prime}\right)$ [19]. The basis $\left\{\tilde{e}_{k}=T e_{k}, \tilde{h}_{k}=T h_{k}\right\}$ has the required properties.

Now we can prove the lemma used in the proof of Proposition 3 .

Lemma 6. Let $L$ be an arbitrary subspace of $Z$. Then there exists a symplectic basis in $Z$ such that $\operatorname{dim} L$ vectors of this basis lie in $L$.

Proof. If the subspace $L$ is either symplectic or isotropic then the assertion of the lemma follows respectively from Lemma 4 (with the remark after it) and Lemma 5 .

If the subspace $L$ is neither symplectic nor isotropic then

$$
L_{1}=L \cap L^{\perp}=\left\{z \in L \mid \Delta\left(z, z^{\prime}\right)=0, \forall z^{\prime} \in L\right\}
$$

is a nontrivial subspace of $L$. Let $L_{2}$ be an arbitrary subspace such that $L=L_{1}+L_{2}$, i.e. $L=L_{1} \vee L_{2}$ and $L_{1} \cap L_{2}=\{0\}$. Then the subspace $L_{2}$ is symplectic. Indeed, if there is a vector $z_{0} \in L_{2}$ such that $\Delta\left(z_{0}, z\right)=0$ for all $z \in L_{2}$ then $\Delta\left(z_{0}, z+z^{\prime}\right)=0$ for all $z^{\prime} \in L_{1}$, $z \in L_{2}$, which implies $z_{0} \in L_{1}$ and hence $z_{0}=0$. 
By Lemma 4 the subspace $L_{2}^{\perp}$ is symplectic. It obviously contains the isotropic subspace $L_{1}$. By Lemma 5 there exists a symplectic basis $\left\{e_{k}, h_{k}\right\}$ in $L_{2}^{\perp}$ such that $\left\{e_{1}, \ldots, e_{d}\right\}$ is a basis in $L_{1}$. By joining this basis and any symplectic basis in $L_{2}$ we obtain a basis with the required properties.

\section{References}

[1] Arnold V.I., Mathematical Methods Of Classical Mechanics (Springer, 1989).

[2] Caruso F., Giovannetti V. "Qubit quantum channels: A characteristic function", Physical Review A 76, 042331 (2007); arXiv:0707.4443.

[3] Caruso F., Eisert J., Giovannetti V., Holevo A.S., "Multi-mode bosonic Gaussian channels", New Journal of Physics 10, 083030 (2008); arXiv:0804.0511.

[4] Caruso F., Eisert J., Giovannetti V., Holevo A.S., "The optimal unitary dilation for bosonic Gaussian channels", Phys. Rev. A 84, 022306 (2011); arXiv:1009.1108.

[5] Chruscinski D., Kossakowski A., "On Partially Entanglement Breaking Channels", Open Sys. Information Dyn., 13, 17-26 (2006); arXiv:quant-ph/0511244.

[6] Demoen B., Vanheuverzwijn P., Verbeure A., "Completely positive quasi-free maps on the CCR algebra", Rep. Math. Phys. 15, 27-39 (1979).

[7] Devetak I., Shor P., "The capacity of a quantum channel for simultaneous transition of classical and quantum information", arXiv:quant-ph/0311131.

[8] Duan R., Severini S., Winter A., "Zero-error communication via quantum channels, noncommutative graphs and a quantum Lovasz theta function", IEEE Trans. Inf. Theory 59:2, 1164-1174 (2013).

[9] Eisert J., Wolf M.M., "Gaussian quantum channels", Quantum Information with Continuous Variables of Atoms and Light, 23-42 (Imperial College Press, London, 2007); arXiv:quant-ph/0505151.

[10] Hiai F., Mosonyi M., Petz D., Beny C., "Quantum f-divergences and error correction", Rev. Math. Phys. 23:7, 691-747; arXiv:1008.2529.

[11] Holevo A.S., "Towards the mathematical theory of quantum communication channels", Probl. Inform. Transm. 8:1, 63-71 (1972).

[12] Holevo A.S., Quantum systems, channels, information. A mathematical introduction (Berlin, DeGruyter, 2012). 
[13] Holevo A.S., "On extreme Bosonic linear channels", arXiv:1111.3552.

[14] Holevo A.S., "One-mode quantum Gaussian channels: structure and quantum capacity", Problems of Information Transmission, 43:1, 3-14 (2007); quant-ph/0607051.

[15] Holevo A.S., "On complementary channels and the additivity problem", Probability Theory and Applications, 51:1, 134-143 (2006); arXiv:quant-ph/0509101.

[16] Ivan J.S., Sabapathy K.K., Simon R., "Operator-sum Representation for Bosonic Gaussian Channels", Phys. Rev. A 84, 042311 (2011); arXiv:1012.4266.

[17] Jencova A., Petz D., "Sufficiency in quantum statistical inference", Commun. Math. Phys. 263, 259-276 (2006); arXiv:math-ph/0412093.

[18] Jencova A., "Reversibility conditions for quantum operations", Rev.Math.Phys. 24, 1250016 (2012); arXiv:1107.0453.

[19] Kostrikin A.I., Manin Yu.I., Linear Algebra and Geometry (CRC, 1989).

[20] Medeiros R.A.C., de Assis F.M. "Quantum zero-error capacity", Int. J. Quant. Inf., 3, 135 (2005).

[21] Nielsen M.A., Chuang I.L., Quantum Computation and Quantum Information (Cambridge University Press, 2000).

[22] Ogawa T., Sasaki A., Iwamoto M., Yamamoto H., "Quantum Secret Sharing Schemes and Reversibility of Quantum Operations", Phys. Rev. A 72, 032318 (2005); arXiv:quant-ph/0505001.

[23] Petz D., "Sufficiency of channels over von Neumann algebras", Quart. J. Math. Oxford Ser. (2) 39:153, 97-108 (1988).

[24] Plenio M.B., Virmani S., "An introduction to entanglement measures", Quantum Inf. Comput., 7, 1-51 (2007); arXiv:quant-ph/0504163.

[25] Shirokov M.E., "Reversibility conditions for quantum channels and their applications", Sbornic:Mathematics, 204:8, 1215-1237 (2013); arXiv:1203.0262.

[26] Shirokov M.E., "On singular Bosonic linear channels", arXiv:1302.6879.

[27] A noiseless channel is unitary equivalent to the channel $\rho \mapsto \rho \otimes \sigma$, where $\sigma$ is a given state; a Bosonic linear channel $\Phi_{K, f}$ is noiseless if its parameters satisfy the conditions: $\operatorname{ker} K$ is a symplectic subspace of $Z_{B}$ (possibly, trivial), $\left.K\right|_{[\operatorname{ker} K]^{\perp}}$ is a symplectic 
trasformation $[\operatorname{ker} K]^{\perp} \rightarrow Z_{A}, f(z)=\phi\left(z_{1}\right)$, where $z_{1}$ is the first component of $z$ corresponding to the decomposition $Z_{B}=\operatorname{ker} K \oplus[\operatorname{ker} K]^{\perp}$ and $\phi$ is a characteristic function of a particular state. 